\title{
Spindle and kinetochore-associated complex subunit 3 (SKA3) promotes stem cell-like properties of hepatocellular carcinoma cells through activating Notch signaling pathway
}

\author{
Shuya Bai ${ }^{1}$, Wei Chen ${ }^{1}$, Mengli Zheng ${ }^{1}$, Xiju Wang ${ }^{1,2}$, Wang Peng ${ }^{1}$, Yuchong Zhao ${ }^{1}$, Yun Wang ${ }^{1}$, \\ Si Xiong ${ }^{1}$, Bin Cheng ${ }^{1}$ \\ ${ }^{1}$ Department of Gastroenterology and Hepatology, Tongji Hospital, Tongji Medical College, Huazhong University of Science and Technology, \\ Wuhan, China; ${ }^{2}$ Department of Digestive Endoscopy, The Affiliated Hospital of Guizhou Medical University, Guiyang, China \\ Contributions: (I) Conception and design: B Cheng, S Xiong, S Bai; (II) Administrative support: X Wang, Y Wang; (III) Provision of study materials \\ or patients: S Bai, W Chen; (IV) Collection and assembly of data: S Bai, M Zheng; (V) Data analysis and interpretation: S Bai, W Peng, Y Zhao; \\ (VI) Manuscript writing: All authors; (VII) Final approval of manuscript: All authors. \\ Correspondence to: Dr. Si Xiong, MD. Department of Gastroenterology and Hepatology, Tongji Hospital, Tongji Medical College, Huazhong \\ University of Science and Technology, 1095 Jiefang Avenue, Wuhan 430030, China. Email: xiongsi2015@126.com; Prof. Bin Cheng, MD. \\ Department of Gastroenterology and Hepatology, Tongji Hospital, Tongji Medical College, Huazhong University of Science and Technology, 1095 \\ Jiefang Avenue, Wuhan 430030, China. Email: b.cheng@tjh.tjmu.edu.cn.
}

Background: Cancer stemness contributes to hepatocellular carcinoma (HCC) initiation, metastasis, drug resistance, and recurrence. The spindle and kinetochore-associated (SKA) complex has been shown to be involved in tumor progression; however, its effects on cancer stem cell-like properties have not yet been examined. This research sought to study each subunit of the SKA complex in HCC systematically.

Methods: Bioinformatic analyses were carried out to examine the expression and clinical data of the SKA complex's each subunit in HCC. The expression of the target genes was detected by quantitative reverse transcription-polymerase chain reaction and Western blot assays. Clone formation and Transwell assays were performed to assess the proliferation and migration abilities of the SKA complex's each subunit. Sphere formation assays and subcutaneous xenograft experiments were performed to investigate the effects of SKA complex subunit 3 (SKA3) on the self-renewal and tumorigenic abilities of HCC.

Results: Each subunit of the SKA complex was highly expressed in HCC, but only SKA complex subunit 1 (SKA1) and SKA3 were associated with the poor overall survival of HCC patients. Additionally, the HCC cells overexpressing SKA3 exhibited increased migration, invasion, proliferation, self-renewal, Sorafenib resistance and tumorigenic abilities. Notch signaling played a vital role in the process by which SKA3 promoted HCC stemness.

Conclusions: SKA3 promotes HCC stem cell-like properties via the Notch signaling pathway. As SKA3 appears to act as a regulator of stemness in HCC, it might be a potential molecular target for HCC.

Keywords: Hepatocellular carcinoma (HCC); spindle and kinetochore-associated complex (SKA complex); spindle and kinetochore-associated complex subunit 3 (SKA3); stem cell-like properties; Notch signaling pathway

Submitted Mar 31, 2021. Accepted for publication Jul 23, 2021.

doi: $10.21037 / \mathrm{atm}-21-1572$

View this article at: https://dx.doi.org/10.21037/atm-21-1572

(c) Annals of Translational Medicine. All rights reserved. 


\section{Introduction}

Hepatocellular carcinoma (HCC) is a highly lethal tumor. With more than 841,000 new cases and 781,000 deaths annually, HCC accounts for $5.7 \%$ of all cancers and $8.2 \%$ of all cancer-related deaths, and constitutes a major health problem worldwide (1). As the vast majority of HCC patients are diagnosed at an advanced stage, surgical treatment is difficult, and many patients have to receive systemic treatment; however, the overall survival rate of patients is poor (2). Additionally, even after surgical treatment, HCC still has a high recurrence and metastasis rate (3). There is increasing evidence that a subpopulation of cancer cells possesses stem cell-like properties, such as self-renewal, treatment resistance, and permanent proliferation abilities, which lead to complex tumor heterogeneity, cancer initiation and development $(4,5)$. However, the mechanism of HCC stemness has not yet been fully elucidated. Clarifying the influencing factors and molecular mechanisms involved in HCC stem cell-like properties, could help develop new therapeutic targets.

Spindle and kinetochore-associated (SKA) complex subunit 1 (SKA1), SKA complex subunit 2 (SKA2), and SKA complex subunit 3 (SKA3) compose the SKA complex, which plays a vital role in the process of precise chromosome separation in cell mitosis $(6,7)$. This key complex stabilizes the kinetochore-microtubule interface through interactions with the $\mathrm{Ndc} 80$ complex, which is comprised of Nuf2, Spc24, and Spc25 $(8,9)$. Welburn showed that the SKA complex binds directly to microtubules (10). Recently, numerous studies have been conducted on the relationship between the SKA complex and cancer. These researches have shown that the SKA complex is related to tumor metastasis and proliferation in various kinds of cancers (11-15). Further, the altered expression of the SKA complex has been shown to mediate various cellular processes in cancer and be associated with poor prognosis of cancer $(16,17)$; however, its role in cancer stem cell-like properties has not yet been explored.

We systematically investigated the role of the whole SKA complex in HCC stemness and its potential mechanism. After examining The Cancer Genome Atlas (TCGA) data sets, we found that each subunit of the SKA complex was up-regulated in HCC, and that SKA1 and SKA3 (but not SKA2) were associated with the poor overall survival of HCC patients. We further examined the effects of SKA1 and SKA 3 on the proliferation and migration in HCC. Our results revealed that SKA3 promoted HCC stem cell-like properties through the activation of the Notch signaling pathway.

This article is presented following the ARRIVE reporting checklist (available at https://dx.doi.org/10.21037/ atm-21-1572).

\section{Methods}

\section{Clinical HCC samples}

All of the samples (primary HCC and their corresponding adjacent non-HCC tissues) were collected from patients undergoing surgical resection at the Tongji Hospital, Huazhong University of Science and Technology (HUST, Wuhan, China). None of the patients received radiotherapy or chemotherapy before surgery. All the samples were histopathologically diagnosed as primary HCC. A small part of the tumorous and adjacent tissues was fixed with $4 \%$ paraformaldehyde for each pair of samples and then embedded in paraffin for immunohistochemistry (IHC) analysis. The rest were stored in an ultra-low temperature refrigerator for later use. Informed consent was obtained from the patients before surgery. The study was conducted following the Declaration of Helsinki (as revised in 2013). The collection and use of human samples were approved by the Ethics Committee of Tongji Hospital, HUST, Wuhan, China (IRB ID: TJ-IRB20210729).

\section{IHC}

After fixing the HCC patients' tumorous and adjacent tissues with 4\% paraformaldehyde, IHC staining with antibodies against SKA3 was performed to detect the protein expression levels. For the mice tumor tissues, IHC staining with antibodies against Ki67 was performed to detect the cell proliferation.

\section{Cell lines and cell culture}

Human-hepatic L02 cell line (KCB Cat\# KCB 200511YJ, RRID: CVCL_6926), Chang-liver cell line [CLS Cat\# 300139/p774_Chang-Liver_(HeLa), RRID: CVCL_0238], and HCC cell lines MHCC-97h (RRID: CVCL_4972) were obtained from the Cell Bank of the Chinese Academy of Sciences (Shanghai, China). SNU-398 (KCLB Cat\# 00398, RRID: CVCL_0077) was purchased from the American Type Culture Collection (ATCC, Manassas, VA, USA), and cultured in RPMI 1640 Medium (GIBCO). 
The other cell lines were cultured in Dulbecco's Modified Eagle's Medium (DMEM; GIBCO). All medium was supplemented with fetal bovine serum (FBS; $10 \%$, GIBCO). The cells were cultured at $37{ }^{\circ} \mathrm{C}[5 \%$ carbon dioxide $\left(\mathrm{CO}_{2}\right)$ condition].

\section{Western blot and antibodies}

Western blot assay was performed as described previously (18). The primary antibodies were as follows: SKA3 (rabbit polyclonal antibody, Abcam Cat\# ab91559, RRID: AB_2049220), Notch1 (Cell Signaling Technology Cat\# 3608, RRID: AB_2153354), cleaved Notch1 (Cell Signaling Technology Cat\# 4147, RRID: AB_2153348), GLI1 (Cell Signaling Technology Cat\# 3538, RRID: AB_1903989), $\beta$-catenin (Cell Signaling Technology Cat\# 8480, RRID: AB_11127855), Hes1 (Cell Signaling Technology Cat\# 11988, RRID: AB_2728766) and Oct4 (Cell Signaling Technology Cat\# 2750, RRID:AB_823583). Anti- $\beta$ actin (Proteintech, 66009-1-ig, 1:5,000) was used as an internal control. The blot density of the protein bands was quantified by the ImageJ software (NIH, America).

\section{$R N A$ extraction and quantitative reverse transcription-polymerase chain reaction (qRT-PCR)}

Following the manufacturer's instructions, the ribonucleic acid (RNA) was extracted using trizol reagent (Invitrogen, Carlsbad, CA, USA). The complementary deoxyribonucleic acid (cDNA) was synthesized by reverse transcription using the Prime Script RT Reagent Kit (Takara, Tokyo, Japan). The qRT-PCR assay was carried out as previously described (19). The differences the between different samples were measured using the $2^{-\Delta \Delta C t}$ method. The SKA3 primer sequence was as follows: sense strand: 5'-TACACGAGCAAGAAGCCATTAAC-3'; and antisense strand: 5'-GGATACGATGTACCGCTCAAGT-3'. The CD44 primer sequence was as follows: sense strand: 5'-CTGCCGCTTTGCAGGTGTA-3'; and antisense strand: 5'-CATTGTGGGCAAGGTGCTATT-3'.

\section{siRNA, plasmids and lentivirus}

The transfection of the small-inhibitory RNA (siRNA) and plasmid was performed using the reagent of Lipofectamine 3000 (Invitrogen, Carlsbad, America) following the instructions of the manufacturer. The sequences of siRNA for SKA3 were as follows: SKA3
siRNA1, 5'-GACCCAGAGTTGTCTAATT-3'; SKA3 siRNA2, 5'-GGTACATCGTATCCCAAGT-3'; SKA3 siRNA3，5'-GAATCCAGGCTCAATGATA-3'. The human full-length SKA3 gene was acquired and inserted into the $\mathrm{pGV}$ plasmid to achieve the over-expression of SKA3. The sequence for lentivirus-based RNA interference (RNAi) targeted SKA3 was as follows: SKA3 shRNA, 5'-GACCCAGAGTTGTCTAATT-3'. The sequence for lentivirus-based RNAi targeted Notch1 was as follows: 5'-GGAGCATGTGTAACATCAA-3'. The knockdown and over-expression of SKA3 were verified by qRT-PCR and Western blot assays.

\section{Colony formation assay}

One thousand cells per well were seeded in 6-well plates. About 2 weeks later, clones were fixed with $4 \%$ methanol for 20 minutes and dyed with crystal violet solution. A light microscope was used to count the clone ( $>50$ cells) numbers.

\section{Cell counting kit-8 (CCK8) and CCK8 toxic assay}

HCC cell proliferation was analyzed CCK8 (Promoter, China). Cells were planted in 96-well plates (1,000 cells/plate) and incubated at $37^{\circ} \mathrm{C}$. Absorption value at $450 \mathrm{~nm}$ was measured at 24, 48, 72, and $96 \mathrm{~h}$, respectively. CCK8 was used to detect the sensitivity of the cells to Sorafenib. After cell adhesion in the 96-well plates, they were treated by Sorafenib (Sigma-Aldrich) at different concentrations $(2.5,5,10$ or $20 \mu \mathrm{M})$. After incubating for $24 \mathrm{~h}$, CCK8 was used to detect the absorbance value.

\section{Transwell migration and invasion assays}

Transwell chambers ( $8 \mu \mathrm{m}$ pore size; Millipore, Billerica, MA, USA) were used to detect the migration and invasion abilities of HCC cells. For the invasion assay, $50 \mathrm{mg} / \mathrm{L}$ Matrigel (1:8 diluent) was added to coat the Transwell chamber surface. Two hundred $\mu \mathrm{L}$ of the cell suspension without FBS was added to the upper chamber, and 10\% FBS culture medium was added as a chemoattractant. The data are shown as mean \pm standard deviation $(\mathrm{SD})$.

\section{Sphere formation assay}

A single-cell suspension was prepared in serum-free DMEM/ F12 medium (cat\# 12400-024; GIBCO, Grand Island, 
NY, USA) with $100 \mu \mathrm{g} / \mathrm{mL}$ streptomycin, $100 \mathrm{IU} / \mathrm{mL}$ penicillin, $20 \mathrm{ng} / \mathrm{mL}$ human recombinant epidermal growth factor (EGF, cat\# PHG0311; GIBCO), $10 \mathrm{ng} / \mathrm{mL}$ human recombinant basic fibroblast growth factor (bFGF, cat\# PHG0266; GIBCO), 2\% B27 supplement (cat\# 17504-044; GIBCO, Grand Island, NY, USA), $1 \% \mathrm{~N}-2$ supplement (cat\# 17502-048; GIBCO, Carlsbad, CA, USA), and 1\% methyl cellulose (cat\# M0262; Sigma-Aldrich). The cells were then seeded in ultra-low attachment 24 -well plates (Corning, NY, USA) at a density of $2 \times 10^{3}$ cells $/ \mathrm{mL}$. Spheres more than $100 \mu \mathrm{m}$ in diameter were counted by a light microscope.

\section{Mouse xenograft tumor assay}

The experiments were approved by the Institutional Animal Care and Use Committee of the HUST (ID: TJH201912001), in compliance with the guidelines on the care and use of animals. We obtained 4-week-old BALB/c nude mice from Beijing Huafukang Biotechnology Company and fed them in pathogen-free conditions. In the subcutaneous xenograft tumor model, $1 \times 10^{6}, 1 \times 10^{5}$ cells were injected subcutaneously. Mice were randomly divided into 4 groups (with 3 mice per group). Mice were monitored for 4 weeks in specific pathogen free (SPF) animal house and examined for the growth of subcutaneous tumors. After being anesthesia, the mice were killed, and the tumors were dissected. Tumor volume was calculated using the following formula: $\mathrm{V}=\mathrm{LW} / 2$, where $\mathrm{L}$ is the length and $\mathrm{W}$ is the width of the tumor. The data are shown as mean \pm SD.

\section{Statistical analysis}

Results were analyzed by the GraphPad Prism 6.0 statistical software. We performed the logistic regression and Wilcoxon signed-rank test to estimate the correlation between SKA3 and HCC clinical-pathological characteristics. To analyze whether SKA3 expression can be regarded as an independent risk factor, we performed univariate and multivariate Cox regression analysis. For comparisons between two groups, Student's $t$-test was used. One-way analysis of variance (ANOVA) test was used for comparisons between more than two groups. All of the analyses were performed using R (http://www.R-project.org, version 3.5.2) or Statistical Product and Service Solutions (SPSS, IBM Corp, Armonk, NY, USA), version 25. The data are expressed as mean $\pm \mathrm{SD} . \mathrm{P}<0.05$ was regarded as significant difference.

\section{Results}

\section{The SKA family was bighly expressed in HCC}

To learn about the contribution of the SKA family to HCC, we downloaded TCGA transcriptome data sets, including 50 normal liver samples and 374 HCC samples. The clinical data of the 374 HCC samples are showed in Figure S1. According to the analysis, the expression of SKA1, SKA2, and SKA3 in HCC were higher in HCC tumour tissues than normal liver samples (see Figure S2A). Among them, the SKA3 expression level in HCC tumour tissues was significantly higher than in normal tissues, and the $\log 2$ [fold change (FC)] is 17.55. Additionally, Kaplan-Meier analysis revealed that high levels of SKA1 and SKA3 were associated with the poor overall survival of patients with HCC, but the association between SKA2 and overall survival was not significant (see Figure S2B). By conducting a pretest study, we found that SKA3 had a greater ability to promote the proliferation and metastasis of HCC cells than SKA1 (see Figure S3); thus, we chose SKA3 for further study.

\section{SKA3 was upregulated in HCC and predicted poor prognosis}

According to the analysis of TCGA LIHC dataset, compare with normal liver samples, the SKA3 expression was significantly increased in HCC samples (see Figure 1A). To examine the expression of SKA3 in HCC tissues, we randomly selected 32 pairs of samples and quantified the protein levels for SKA3 in HCC tissues and adjacent normal liver tissues. $\beta$-actin was used as the housekeeping gene for confirmation. The protein level of SKA3 in HCC tissues $(\mathrm{T})$ was higher than that in non-tumor tissues $(\mathrm{N})$ (see Figure $1 B, 1 C$ ). The qRT-PCR revealed that SKA3 was more highly expressed in HCC cell lines than normal liver cell lines (see Figure 1D). We examine the expression of SKA3 by IHC staining in HCC tissues and adjacent normal liver tissues. The results showed that the expression of SKA3 was overexpressed in HCC tissues compared with adjacent normal liver tissues (see Figure 1E).

Next, we investigated the correlation between SKA3 expression and clinical-pathological characteristics. The results suggested that a higher SKA3 level was correlated with a higher grade $(\mathrm{P}=1.723 \mathrm{e}-10)$, higher stage $(\mathrm{P}=0.002)$, and larger tumor size $(\mathrm{T})(\mathrm{P}=0.002)$ (see Figure $1 F-1 H)$. Using the logistic regression analysis, we found that SKA3 level was one of the categorical dependent variables (according to the median value of expression) related to 
A

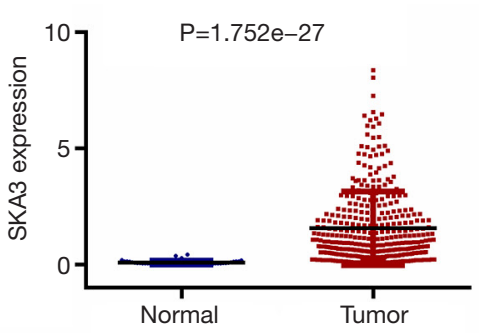

C

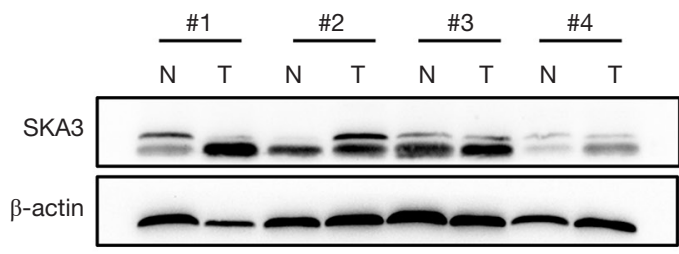

B

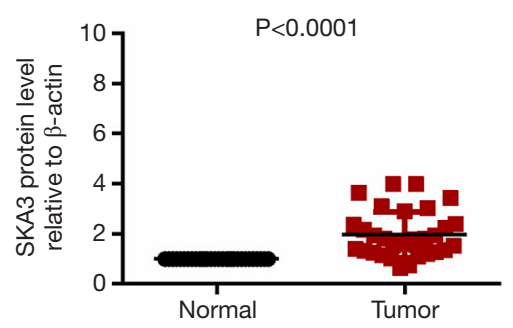

D

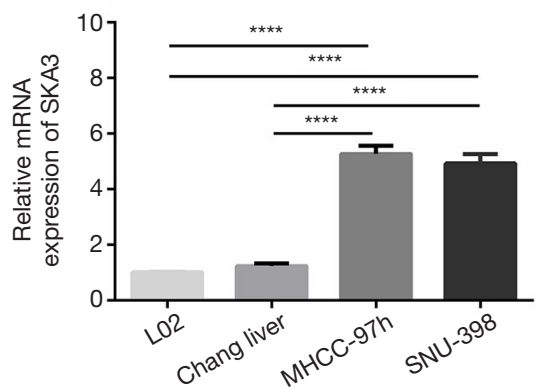

E

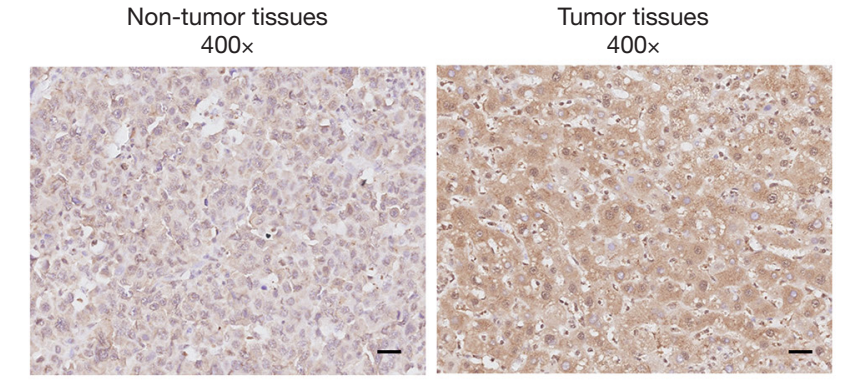

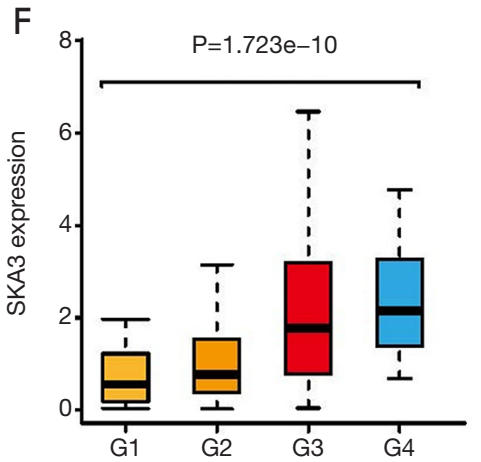

G

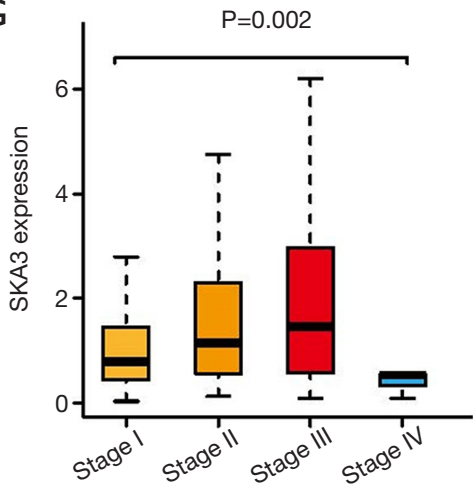

$\mathrm{H}$

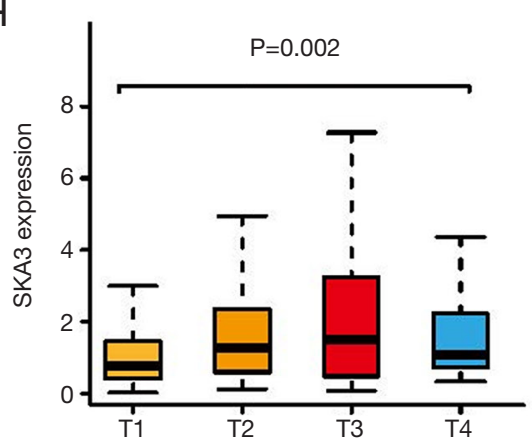

Figure 1 SKA3 was upregulated in HCC and predicted poor prognosis in HCC patients. (A) Bioinformatic analysis of the SKA3 expression level in normal liver samples and HCC samples, including 50 normal liver samples and 374 HCC samples. (B,C) Western blot analysis of SKA3 protein levels in HCC tissues and adjacent non-tumor tissues selected randomly ( $\mathrm{n}=32 ; \mathrm{P}<0.0001$, Student's $t$-test). $\beta$-actin was used as a normalized control. (D) Relative expression levels of SKA3 in HCC cell lines (MHCC-97h, and SNU-398), L02 cells, and Chang-liver cells were measured by using qRT-PCR. (E) Representative images of IHC staining of SKA3 in tumor and adjacent non-tumor tissues (scale bar: $50 \mu \mathrm{m}$ ). (F-H) The relationship between SKA3 expression and grade, stage, tumor size (T) in TCGA. For the statistical analysis (Student's $t$-test), ${ }^{* * * *}, \mathrm{P}<0.0001$. HCC, hepatocellular carcinoma; IHC, immunohistochemistry; qRT-PCR, quantitative reverse transcriptionpolymerase chain reaction; SKA3, spindle and kinetochore-associated complex subunit 3; TCGA, The Cancer Genome Atlas. 
Table 1 The expression ${ }^{\dagger}$ of SKA3 related to clinicopathological features (logistic regression)

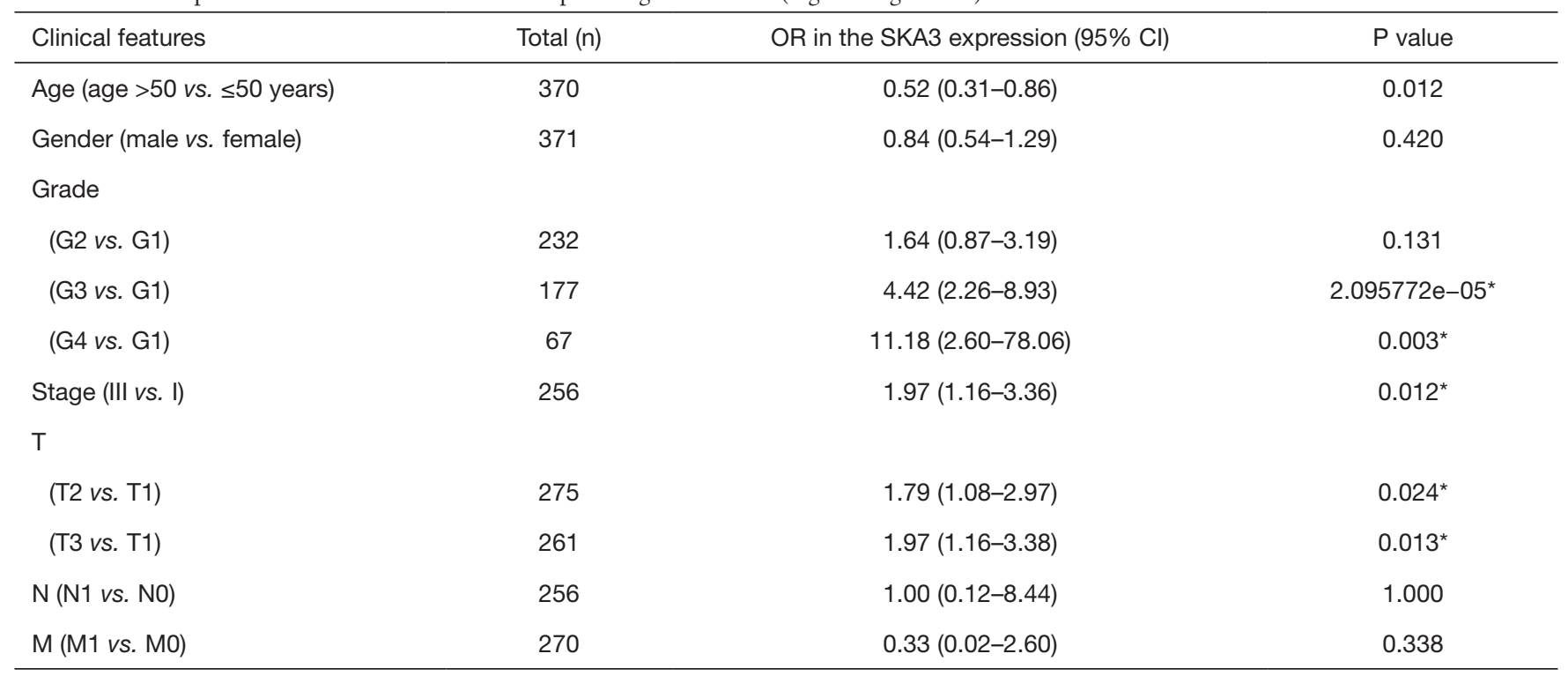

${ }^{*}, \mathrm{P}<0.05 ;{ }^{\dagger}$, categorical dependent variable, greater or less than the median expression level. Cl, confidence interval; OR, odds ratio; SKA3, spindle and kinetochore-associated complex subunit 3.

Table 2 Associations of overall survival with clinicopathological features in TCGA patients (Cox regression)

\begin{tabular}{|c|c|c|c|c|}
\hline Clinicopathological features & \multicolumn{2}{|c|}{ Univariate analysis } & \multicolumn{2}{|c|}{ Multivariate analysis } \\
\hline Age & $1.01(0.99-1.02)$ & 0.591 & $1.01(0.99-1.04)$ & 0.15 \\
\hline Gender (male vs. female) & $0.78(0.49-1.25)$ & 0.301 & $1.03(0.61-1.74)$ & 0.91 \\
\hline Grade & $1.02(0.75-1.39)$ & 0.914 & $1.00(0.72-1.39)$ & 0.99 \\
\hline T classification & $1.80(1.43-2.27)$ & $4.73 \mathrm{e}-07^{*}$ & $1.81(0.77-4.27)$ & 0.18 \\
\hline M classification & $3.85(1.21-12.18)$ & $0.023^{*}$ & $2.54(0.64-10.03)$ & 0.18 \\
\hline $\mathrm{N}$ classification & $2.02(0.49-8.28)$ & 0.328 & $2.35(0.37-14.90)$ & 0.36 \\
\hline SKA3 expression (high vs. low) & $2.05(1.53-2.74)$ & $1.24 \mathrm{e}-06^{*}$ & $2.07(1.52-2.82)$ & $4.38 \mathrm{e}-06^{*}$ \\
\hline
\end{tabular}

*, $\mathrm{P}<0.05$. $\mathrm{Cl}$, confidence interval; HR, hazard ratio; SKA3, spindle and kinetochore-associated complex subunit 3; TCGA, The Cancer Genome Atlas.

pathological features (see Table 1). The results indicated that HCC with a higher SKA3 level tended to have a worse grade, stage, and $\mathrm{T}$ classification. The multivariate and univariate Cox regression analyses revealed that among patients with HCC, SKA3 level was an independent risk factor of overall survival [hazard ratio $(\mathrm{HR})=2.07$, $\mathrm{P}=4.38 \mathrm{e}-06$; see Table 2].

Together, these results indicated that SKA3 expression was significantly up-regulated in HCC samples and was related to poor clinical outcomes in patients with HCC.

\section{SKA3 promoted HCC cell migration and invasion}

SKA3 expression was downregulated by siRNA in MHCC97h and SNU-398 cell lines, and the transfection efficiency was detected by qRT-PCR and Western blot. The results showed that siSKA3-1 had the highest downregulation efficiency (see Figure 2A). In the following article, we will use siSKA3 instead of siSKA3-1. pGV plasmids were used to upregulate SKA3 expression in MHCC-97h and SNU398 cell lines, and the transfection efficiency was also 
A
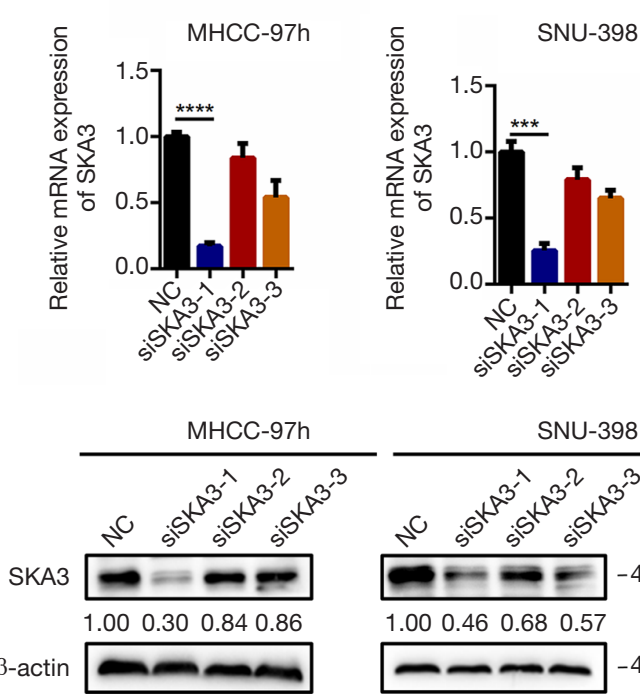

C
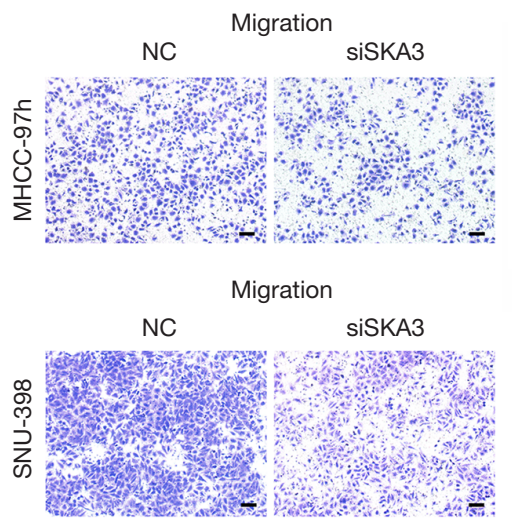

E
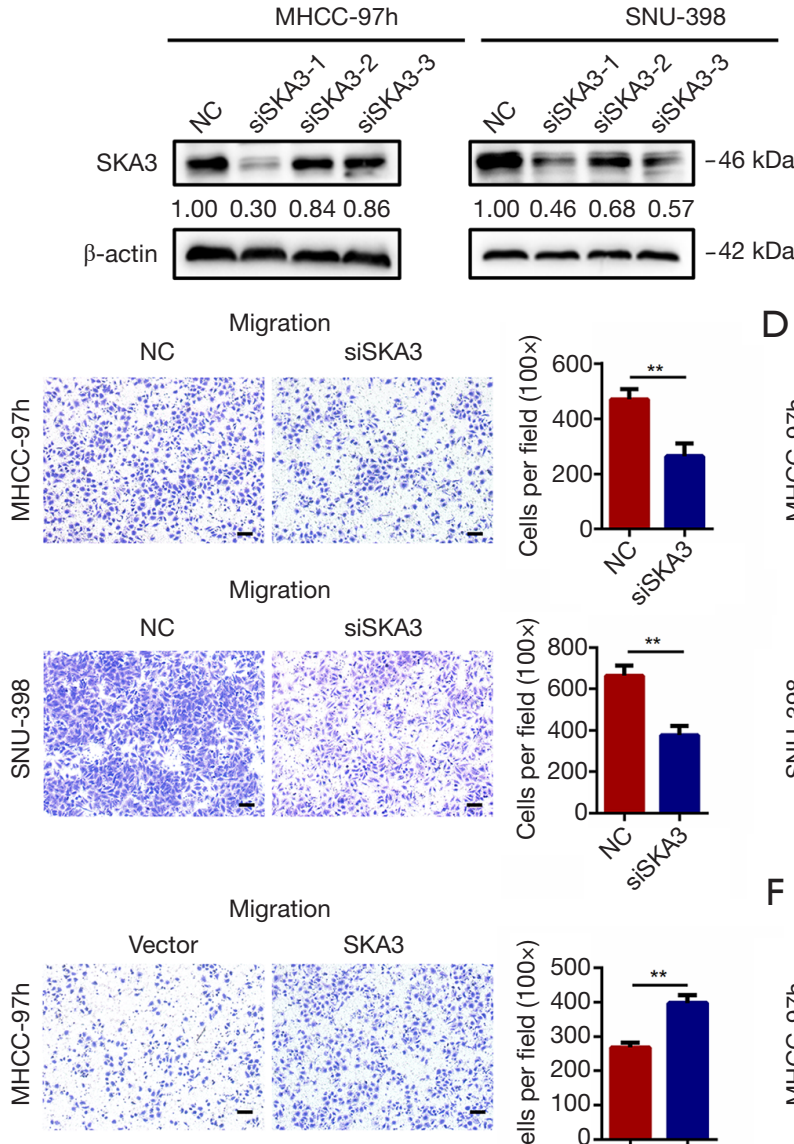

$\mathrm{D}$
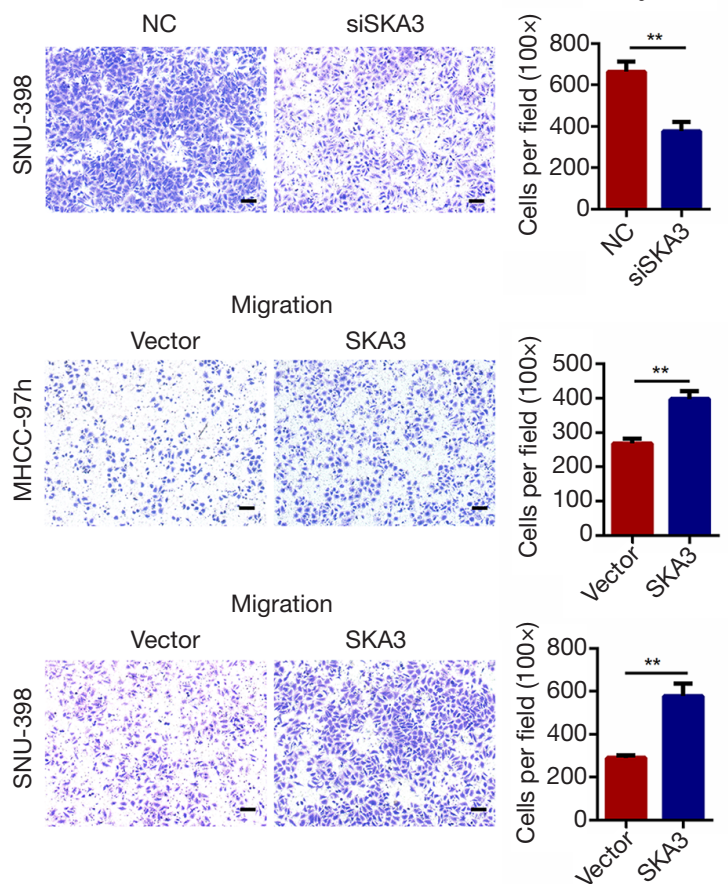

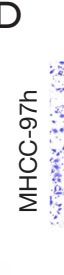

B
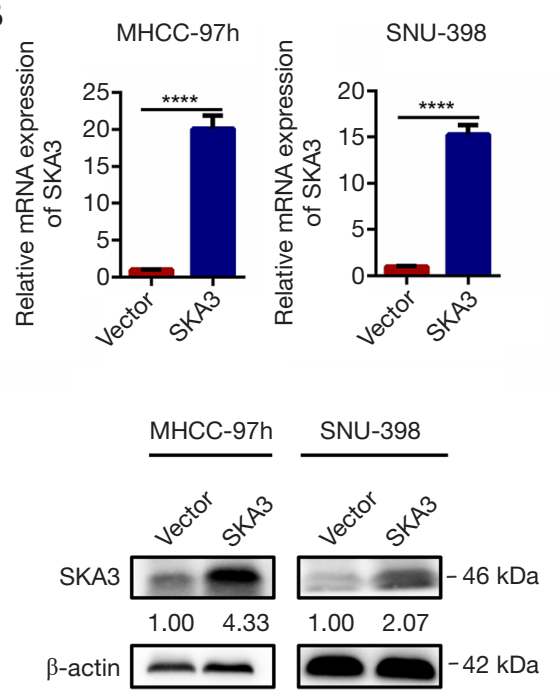

Invasion
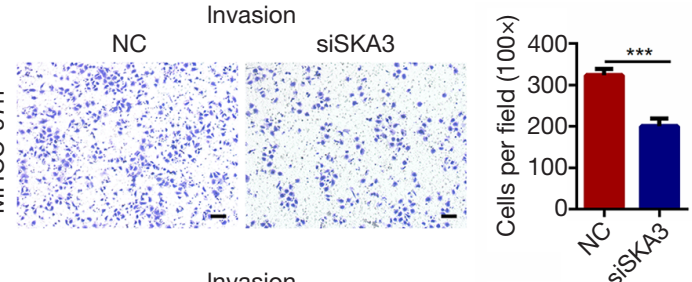

Invasion
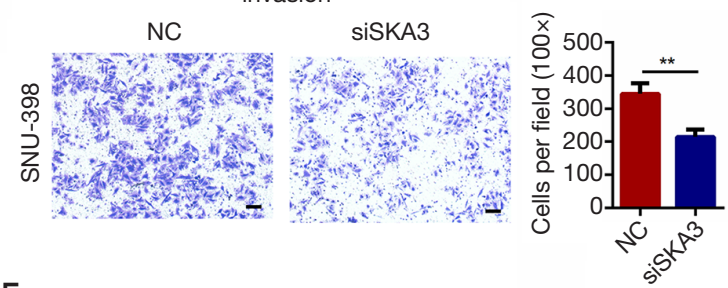

F
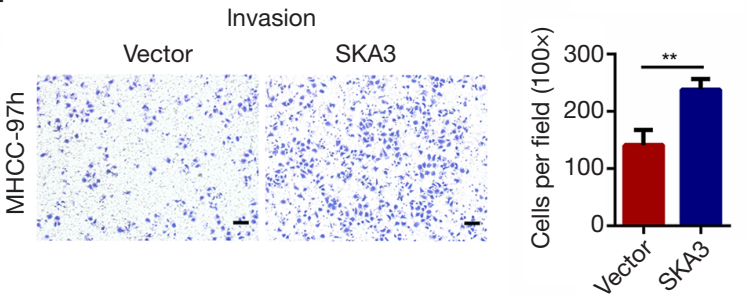

Invasion

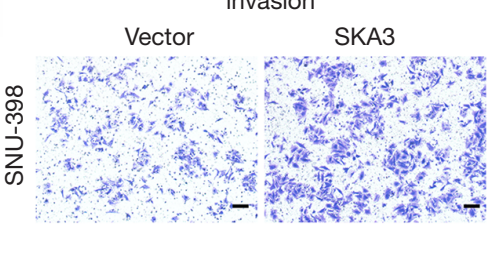

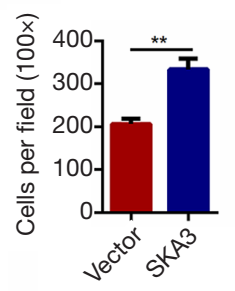

Figure 2 SKA3 promoted HCC cell migration and invasion. (A,B) The selection of siRNA sequences and the regulation of SKA3 in HCC cell lines. qRT-PCR and Western blot assays were used to detect transfection efficiency. (C,D) Representative images of Transwell migration and invasion in siSKA3 MHCC-97h and SNU-398 cells staining with crystal violet solution (scale bar: $200 \mu \mathrm{m})$. (E,F) Representative images of Transwell migration and invasion in SKA3 MHCC-97h and SNU-398 cells staining with crystal violet solution (scale bar: $200 \mu \mathrm{m})$. For the statistical analysis (Student's $t$-test), ${ }^{* *}, \mathrm{P}<0.01$; ${ }^{* * *}, \mathrm{P}<0.001$; ${ }^{* * *}, \mathrm{P}<0.0001$. HCC, hepatocellular carcinoma; qRT-PCR, quantitative reverse transcription-polymerase chain reaction; SKA3, spindle and kinetochore-associated complex subunit 3. 
detected by qRT-PCR and Western blot (see Figure 2B).

We carried out Transwell migration and invasion assays to investigate the effects of SKA3 on the migration and invasion ability of HCC cells. The number of migratory cells and invasion cells decreased when SKA3 was downregulated (see Figure 2C,2D). Conversely, the number of migratory cells and invasion cells increased when SKA3 was upregulated (see Figure 2E,2F).

\section{SKA3 promoted HCC cell proliferation and protected HCC cells from apoptosis}

The colony-forming assay showed that the down-regulation of SKA3 weakened cell proliferation and colony-forming ability (see Figure $3 A$ ). The CCK 8 assay showed that suppressing SKA3 decreased cell viability and proliferation ability (see Figure 3B). Conversely, over-expression of SKA3 had the opposite effects (see Figure 3C,3D). Thus, the downregulation of SKA3 was found to decrease HCC cell proliferation capacity, while the upregulation of SKA3 was found to increase HCC proliferation ability. Next, we conducted an apoptosis assay to test the effect of SKA3 in vitro. Using annexin $\mathrm{V}$ and propidium iodide (PI) staining, we found that the cells downregulating SKA3 had a higher apoptosis rate (see Figure $3 E$ ) and that the upregulation of SKA3 inhibited cell apoptosis (see Figure 3F). Together, these data indicated that SKA3 promoted HCC cell proliferation and protected HCC cells from apoptosis.

\section{SKA3 promoted stem cell-like properties of HCC cells}

Previous research has suggested that SKA3 promotes HCC cell metastasis, invasion, proliferation and inhibit apoptosis (15). To study the relationship between SKA3 and HCC stemness, we analyzed the correlations among SKA3 and stemness markers in TCGA at the GEPIA website (http://gepia. cancer-pku.cn/). The results showed that SKA3 had positive correlations with CD44 and Oct4 in HCC (see Figure 4A). As CD44 and Oct4 are well-accepted stemness markers (20,21), the above data suggested that SKA3 plays a role in regulating HCC stemness.

We then investigated the effects of SKA3 on the stem cell-like properties of HCC. Sphere formation assays were performed to evaluate the self-renewal ability of HCC cells. The over-expression of SKA3 in SNU-398 cells led to more and larger spheres, while the suppression of SKA3 in SNU398 cells led to the formation of fewer and smaller spheres (see Figure 4B). Next, different concentrations of Sorafenib were used to treat MHCC-97h and SNU-398 cells for $24 \mathrm{~h}$. The results showed that cells down-regulating SKA3 were less resistant to Sorafenib, while cells up-regulating SKA3 were more resistant to Sorafenib (see Figure 4C). Western blot and qRT-PCR suggested that inhibiting SKA3 expression suppressed the expression of Oct4 and CD44 in MHCC-97h and SNU-398, while overexpressing SKA3 increased the expression of Oct4 and CD44 (see Figure 4D and Figure S4). Finally, the tumor-initiating capacity of HCC cells was evaluated using a subcutaneous xenograft tumor model in the BALB/c nude mice. MHCC-97h cells were used to establish stable cell lines, shSKA3-MHCC97h cells and SKA3-MHCC-97h cells with lentivirus infection (see Figure 4E). The grouping and treatment of animal experiments are shown in Table 3. An analysis of 3 mice in each group showed that the knockdown of SKA3 decreased the tumor growth of MHCC-97h cells, while the overexpression of SKA3 increased the tumor growth of MHCC-97h cells, which was further confirmed by IHC staining for Ki67 in the xenograft tumors of different groups (see Figure 4F-4H). Thus, these results confirmed that SKA3 enhanced the tumorigenic capacity of HCC cells.

\section{SKA3 promoted stem cell-like properties through the Notch signaling pathway}

Through the previous experiments, we demonstrated that SKA3 displayed marked tumor promoting effects in vitro and in vivo, and promoted stem cell-like properties, such as metastasis, invasion, proliferation, self-renewal, resistance to Sorafenib, and tumorigenic abilities.

To decipher the underlying mechanism of SKA3 in regulating stem cell-like properties, we evaluated 3 major signaling pathways critical for stem cell-like properties; that is, the Hedgehog (22), Notch (23), and Wnt signaling pathways (24). First, we tested the expressions of GLI1, NICD, and $\beta$-catenin, which are the key factors in the Hedgehog, Notch and Wnt signaling pathways, respectively. As Figure $5 A$ shows, after the down-regulation of SKA3, the expression of NICD (but not GLI1 and $\beta$-catenin) decreased. In TCGA, the regression analysis about HCC clinical-pathology showed that SKA3 was positively related to Notch 1 at GEPIA website (see Figure 5B). As stated above, we examined whether SKA3 promoted HCC stem cell-like properties via the Notch signaling pathway. Western blot suggested that inhibiting SKA3 expression significantly suppressed Notch signaling in MHCC-97h and SNU-398, while overexpressing SKA3 
A

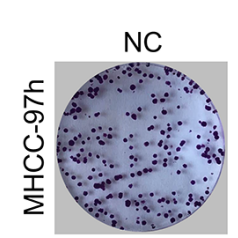

NC

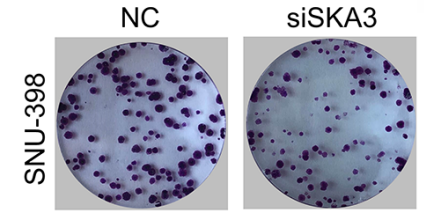

C
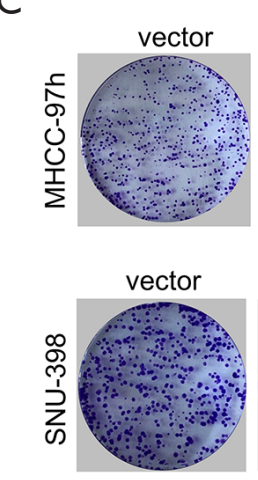

E

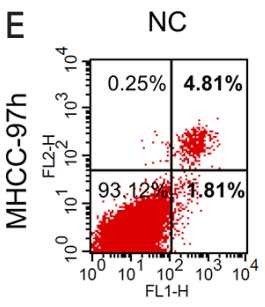

NC

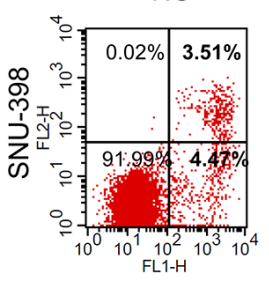

siSKA3

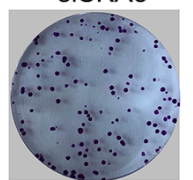

SiSKA3

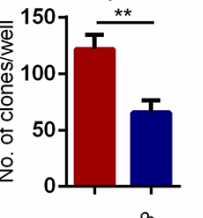

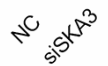

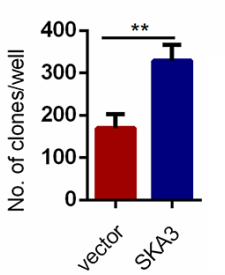

SKA3
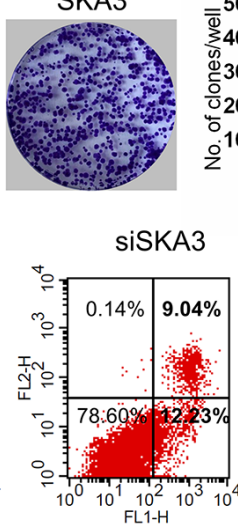

SiSKA3
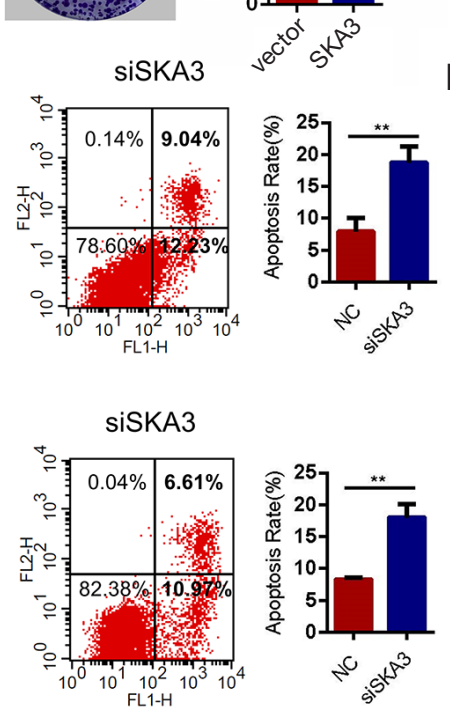

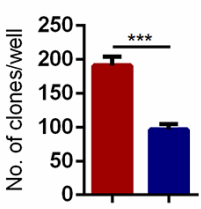

$\div 0^{4 x^{3}}$

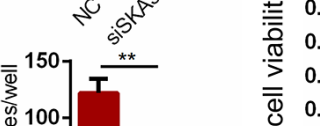

$\bar{\Phi}$

0.0
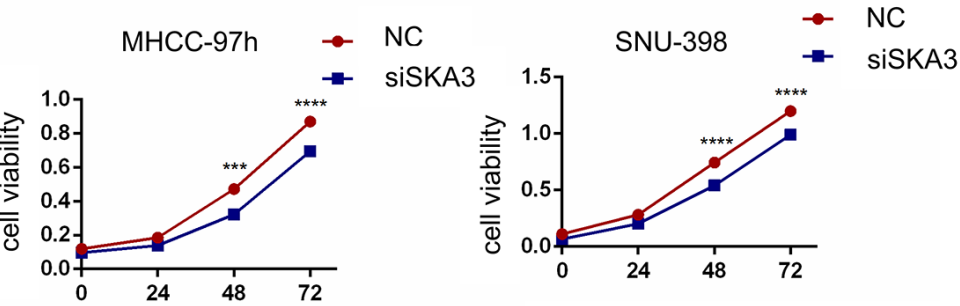

D
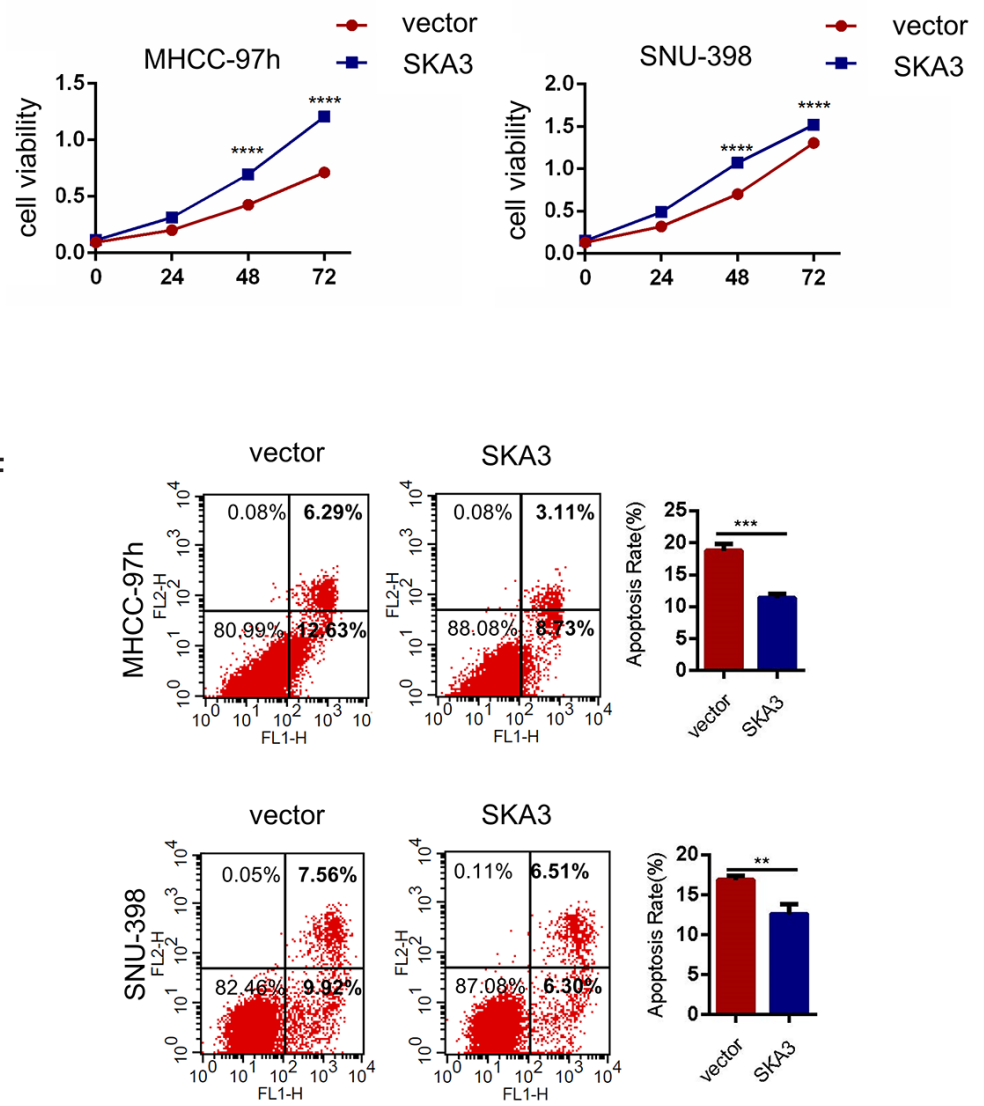

Figure 3 SKA3 promoted HCC cell proliferation and protected HCC cells from apoptosis. (A) Colony formation assay was performed using siSKA3 MHCC-97h and SNU-398 cells. The crystal violet was used for dyeing. (B) CCK8 assay was performed using siSKA3 MHCC-97h and SNU-398 cells. (C) Colony formation assay was performed using SKA3 MHCC-97h and SNU-398 cells. The crystal violet was used for dyeing. (D) CCK8 assay was performed using SKA3 MHCC-97h and SNU-398 cells. (E,F) Apoptosis was assessed by flow cytometry in indicated cells. Cells were treated with annexin $\mathrm{V}$ (an indicator of apoptosis) and propidium iodide. For the statistical analysis (Student's $t$-test), ${ }^{* *}, \mathrm{P}<0.01$; ${ }^{* * *}, \mathrm{P}<0.001$; ${ }^{* * *}, \mathrm{P}<0.0001$. CCK8, cell counting kit-8; HCC, hepatocellular carcinoma; SKA3, spindle and kinetochoreassociated complex subunit 3 . 
A
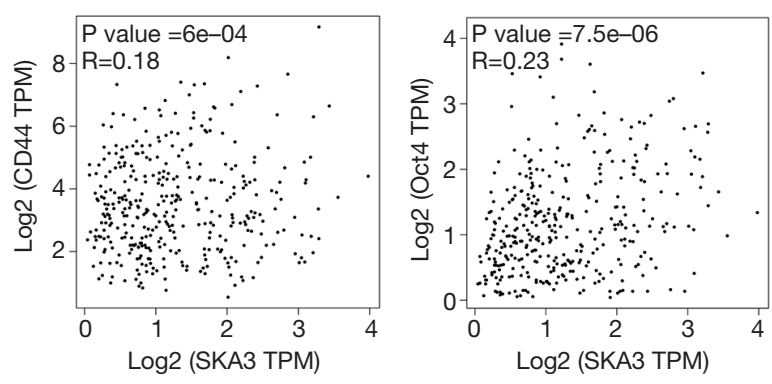

C
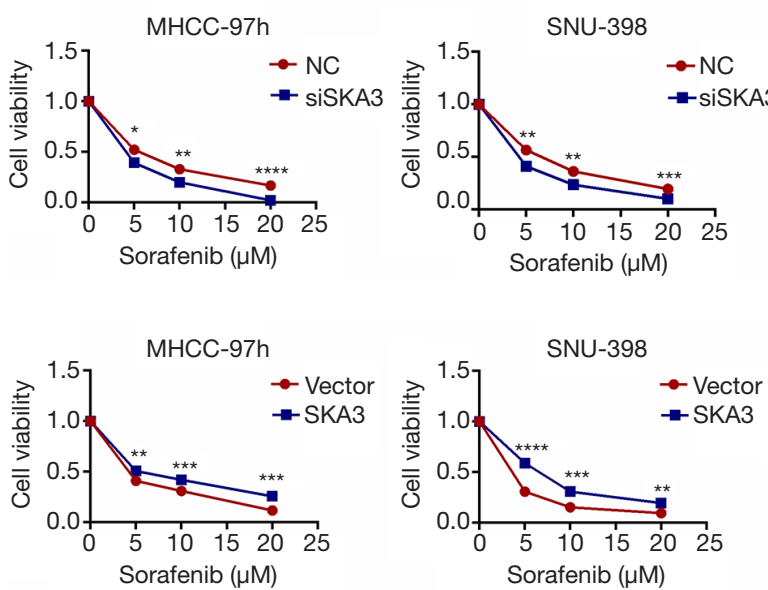
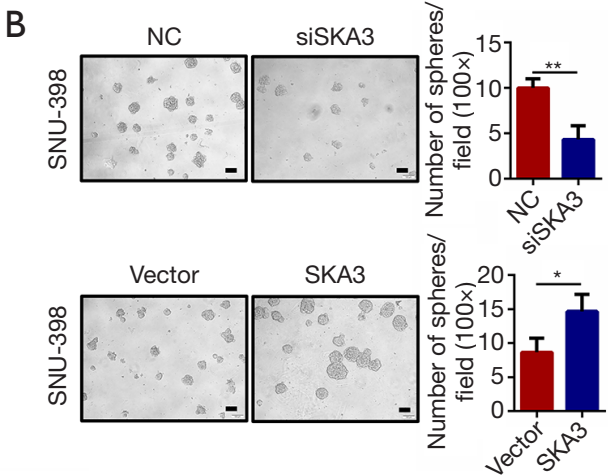

D

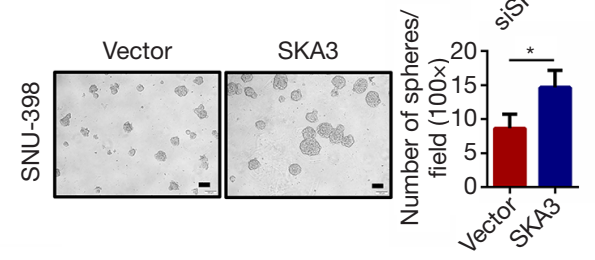

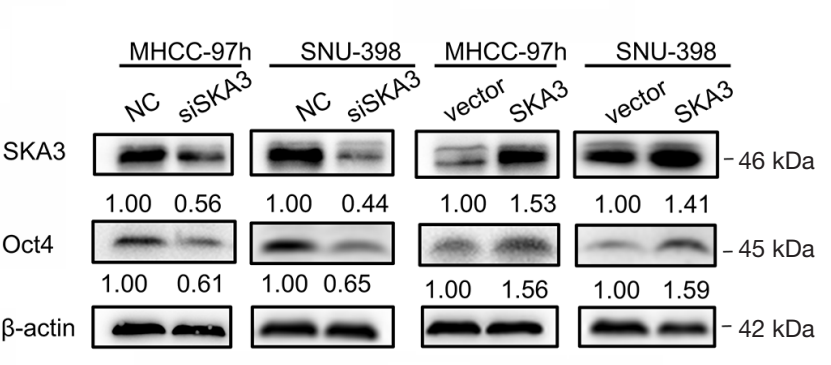

E
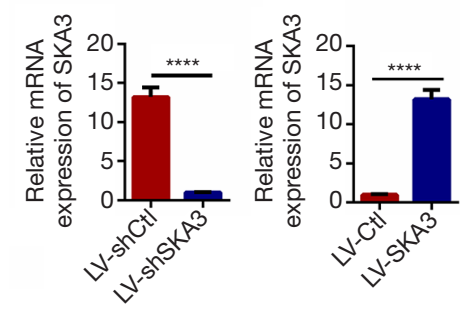

F
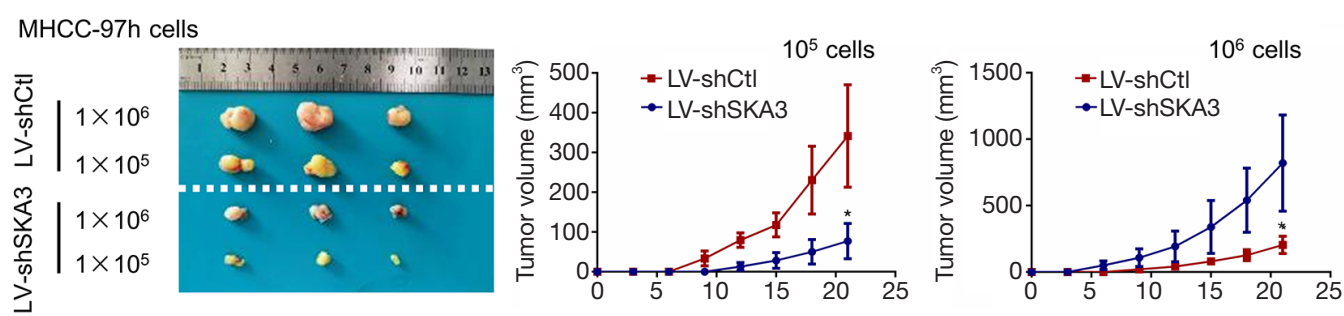

G
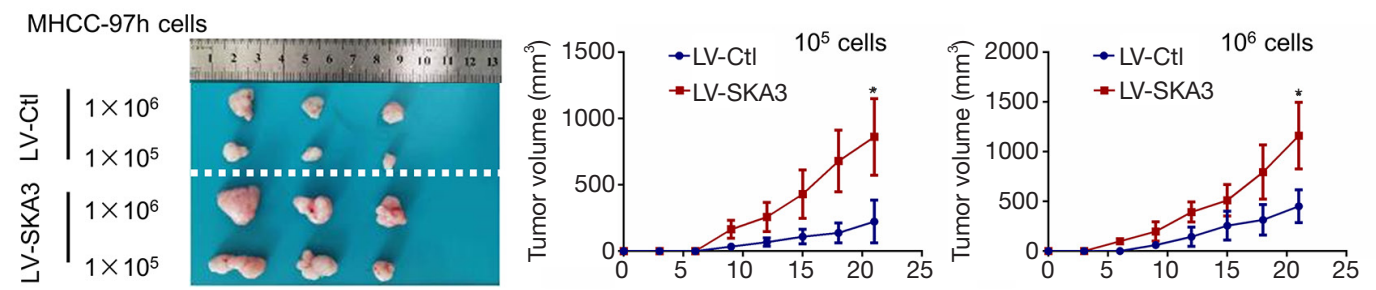
$\mathrm{H}$

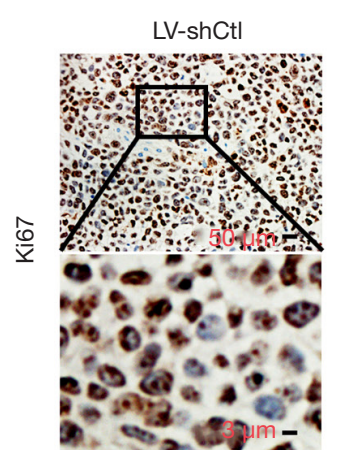

LV-shSKA3

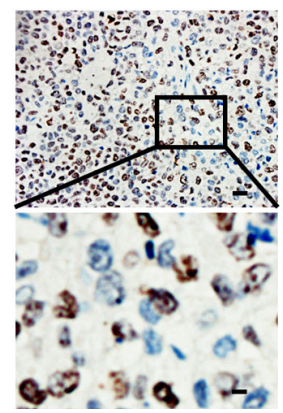

LV-CtI

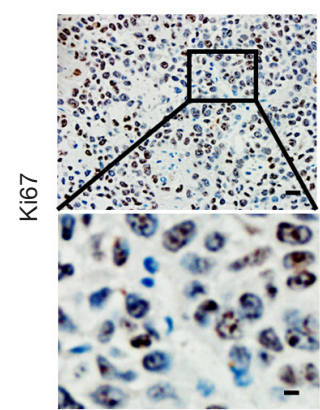

LV-SKA3

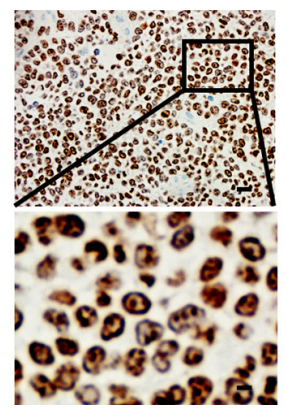

Figure 4 SKA3 promoted HCC stem cell-like properties. (A) Correlations among SKA3 and stemness markers in TCGA HCC tumor are denoted with Pearson's correlation coefficients. (B) Representative images of spheres and histogram analysis in siSKA3 and SKA3 SNU-398 cells (scale bar: $200 \mu \mathrm{m}$ ). (C) siSKA3 cells and SKA3 cells were treated with Sorafenib for $24 \mathrm{~h}$ and evaluated by CCK8 toxic assay. (D) The expression of Oct4 were tested by Western blot in indicated cells. $\beta$-actin was used as the loading control. (E) MHCC-97h cells were used to establish stable cell lines. (F) The efficiency of tumor formation of LV-shCtl and LV-shSKA3 MHCC-97h cells (number of injected cells: $1 \times 10^{6}$, $\left.1 \times 10^{5} ; \mathrm{n}=3\right)$. (G) Efficiency of tumor formation of LV-Ctl and LV-SKA3 MHCC-97h cells (number of injected cells: $\left.1 \times 10^{6}, 1 \times 10^{5} ; \mathrm{n}=3\right)$. $(\mathrm{H})$ The expression of Ki67 determined by IHC in tumors from subcutaneous xenografts in the above groups. For the statistical analysis (Student's $t$-test), ${ }^{*}, \mathrm{P}<0.05$; **, $\mathrm{P}<0.01$; **, $\mathrm{P}<0.001$; ${ }^{* * *}, \mathrm{P}<0.0001$. CCK8, cell counting kit-8; HCC, hepatocellular carcinoma; IHC, immunohistochemistry; SKA3, spindle and kinetochore-associated complex subunit 3; TCGA, The Cancer Genome Atlas; TPM, transcript per million.

Table 3 Characteristics and health status of animals and the treatment to them

\begin{tabular}{|c|c|c|c|}
\hline Mouse & Weight (g) (4 weeks old) & Microbiological status & Injected cells \\
\hline $1 / 2 / 3$ & $12.3 / 13.1 / 15.2$ & Sterile & LV-shCtl $\left(1 \times 10^{6}\right)$ \\
\hline $4 / 5 / 6$ & $13.5 / 13.9 / 15.7$ & Sterile & LV-shSKA3 $\left(1 \times 10^{6}\right)$ \\
\hline $7 / 8 / 9$ & $12.7 / 15.3 / 16.1$ & Sterile & LV-shCtl $\left(1 \times 10^{5}\right)$ \\
\hline $10 / 11 / 12$ & $14.1 / 14.7 / 16.9$ & Sterile & LV-shSKA3 $\left(1 \times 10^{5}\right)$ \\
\hline $16 / 17 / 18$ & 12.9/16.7/17.1 & Sterile & LV-SKA3 $\left(1 \times 10^{6}\right)$ \\
\hline $19 / 20 / 21$ & $13.8 / 15.6 / 17.7$ & Sterile & LV-Ctl $\left(1 \times 10^{5}\right)$ \\
\hline $22 / 23 / 24$ & $14.5 / 17.2 / 18.0$ & Sterile & LV-SKA3 $\left(1 \times 10^{5}\right)$ \\
\hline
\end{tabular}

SKA3, spindle and kinetochore-associated complex subunit 3.

activated Notch signaling (see Figure 5C). Notably, after we knocked down the expression of Notch1 in SKA3 cells via lentivirus transfection, SKA3-mediated HCC migration, proliferation, self-renewal, and resistance to Sorafenib capacities were remarkably inhibited (see Figure 6). Thus, SKA3 was found to promote HCC stem cell-like properties by activating the Notch signaling pathway.

\section{Discussion}

The stemness of cancer is considered as the "source power" of tumorigenesis and development. There is increasing evidence that due to the existence of liver cancer stem cells, the risk of tumorigenic, metastasis, drug resistance, and recurrence of $\mathrm{HCC}$ is increased (25). For example, cancer stem cells expressing high epithelial cell adhesion molecule (EpCAM) are thought to be responsible for the initiation of tumor progression, metastasis, drug resistance, and recurrence (26). Thus, it is necessary to explore the factors and molecular mechanisms that regulate the stem cell-like properties of HCC. In this study, we found that SKA3, one subunit of the SKA complex, was highly expressed in HCC 

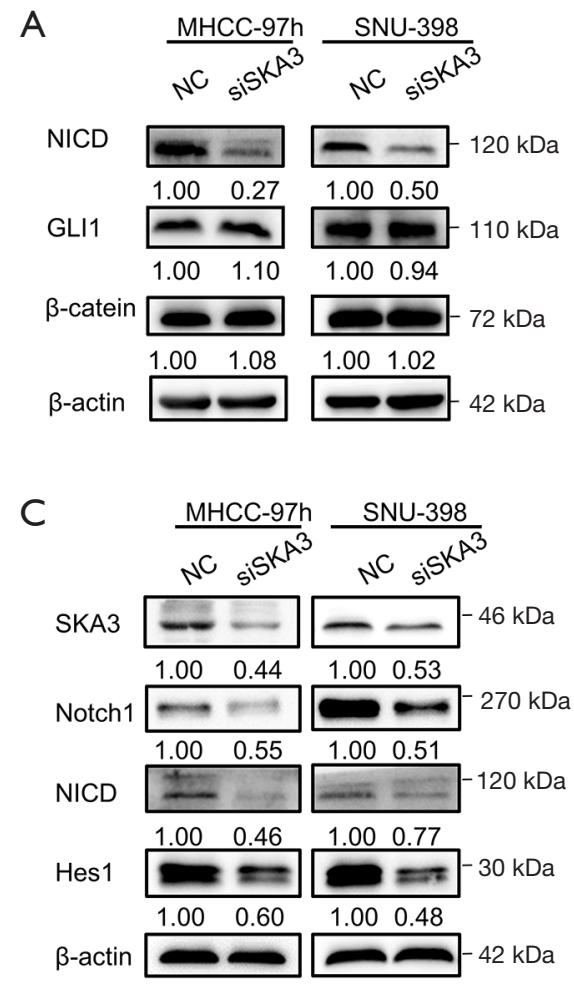
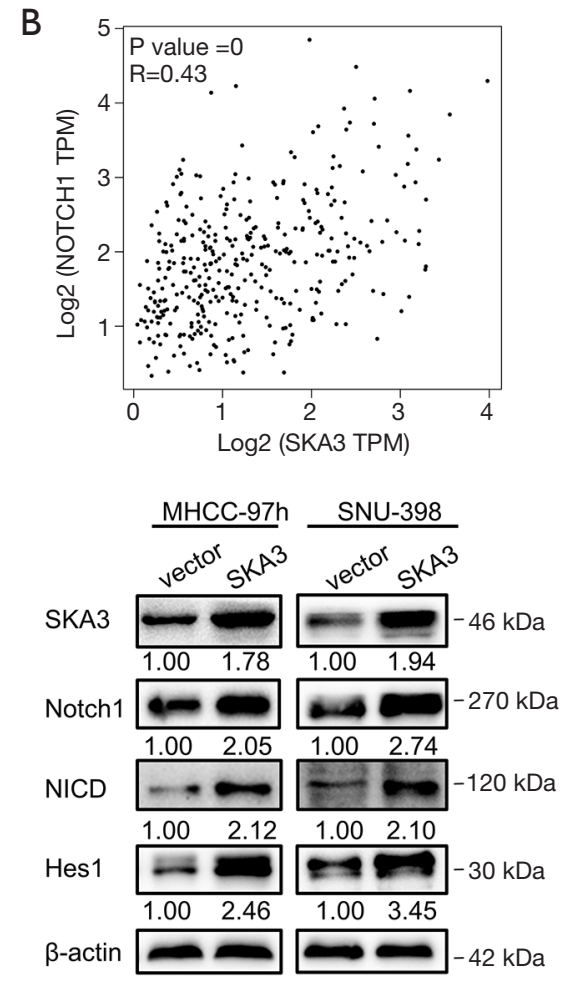

Figure 5 SKA3 activated the Notch signaling pathway in HCC cells. (A) Western blot analysis of featured protein levels of Notch, Hedgehog and Wnt pathways in siSKA3 cells. $\beta$-actin was used as the loading control. (B) Correlations among Notch1 and SKA3 in TCGA HCC tumor are denoted with Pearson's correlation coefficients. (C) The core components of Notch signaling, including Notch1 receptor, NICD and Hes1 were tested in siSKA3 and SKA3 MHCC-97h and SNU-398 cells by Western blot. $\beta$-actin was used as a normalized control. HCC, hepatocellular carcinoma; SKA3, spindle and kinetochore-associated complex subunit 3; TCGA, The Cancer Genome Atlas; TPM, transcript per million.

A
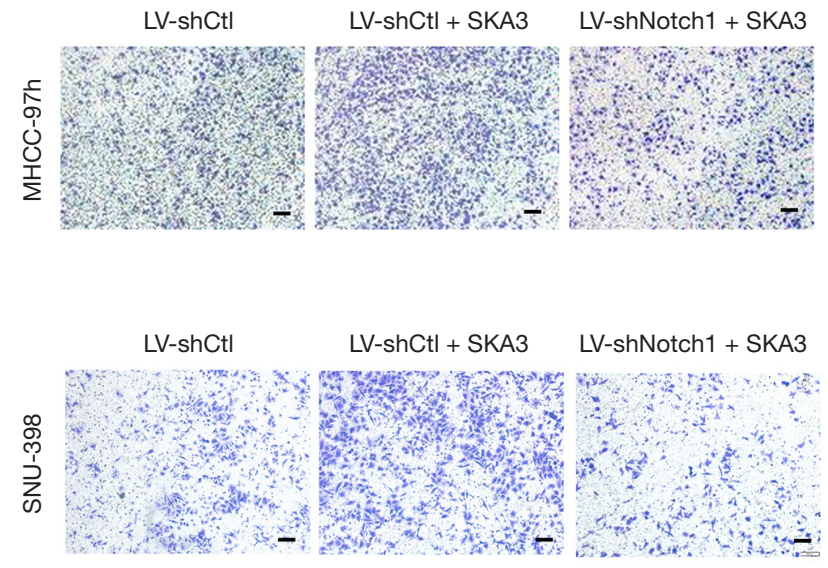

MHCC-97h

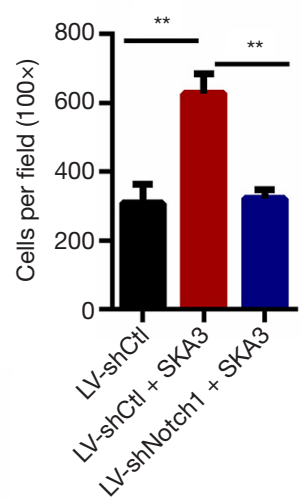

SNU-398

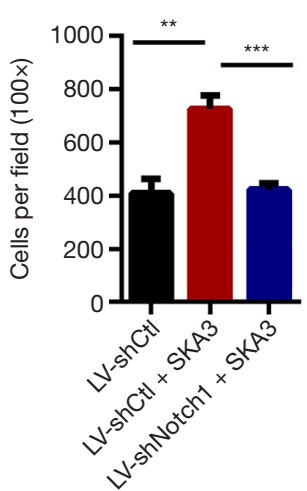


B
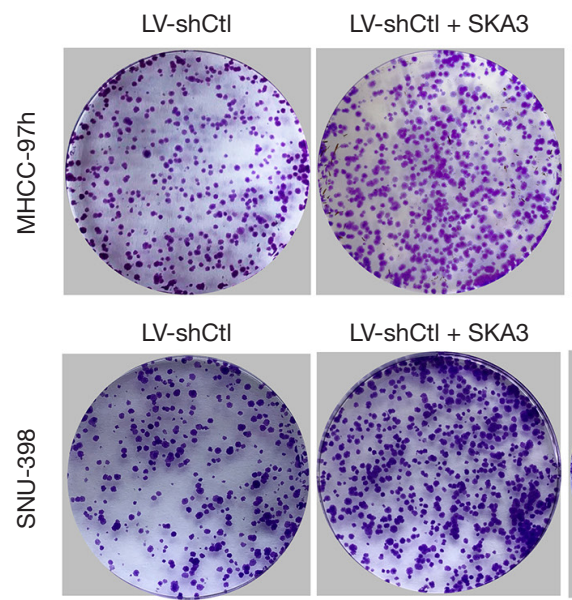

LV-shCtI + SKA3
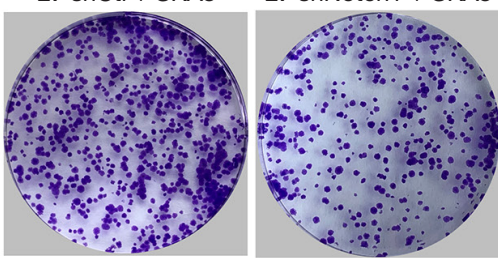

C
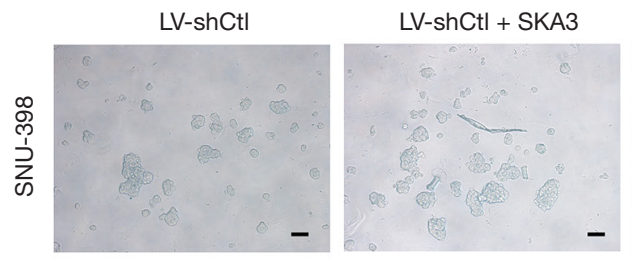

LV-shNotch1 + SKA3

D

MHCC-97h

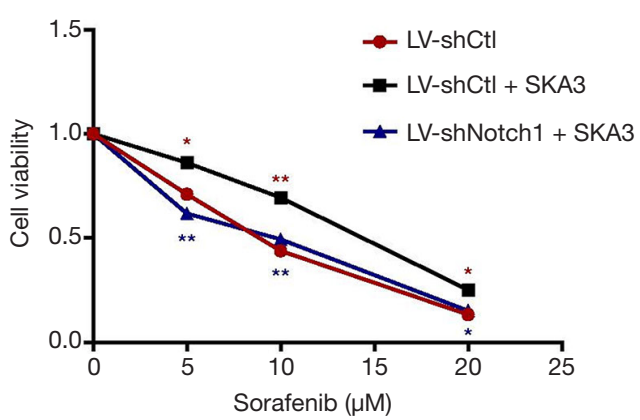

LV-shNotch1 + SKA3

LV-shNotch1 + SKA3
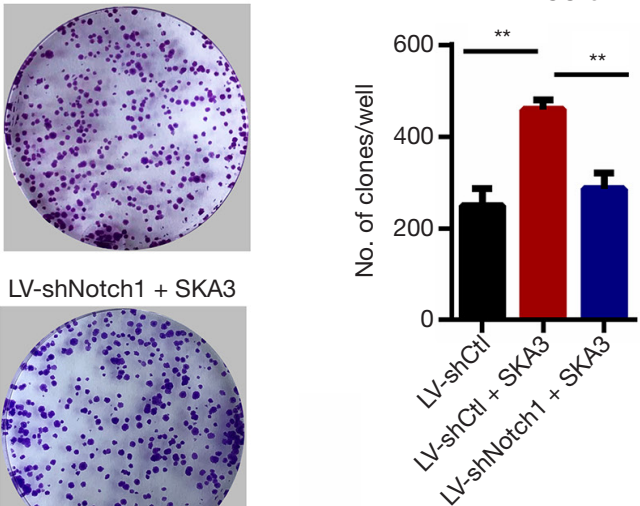

SNU-398

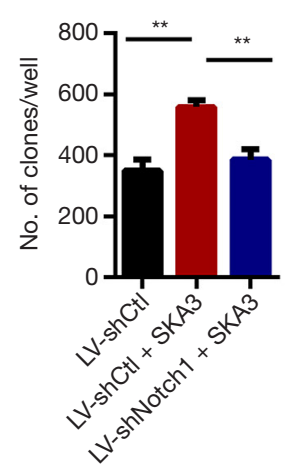

SNU-398

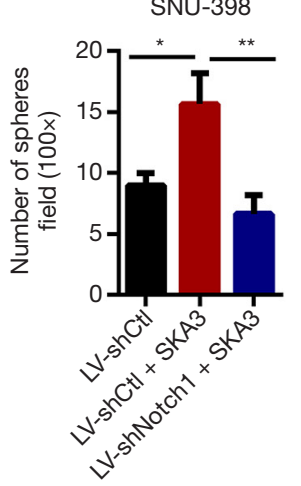

SNU-398

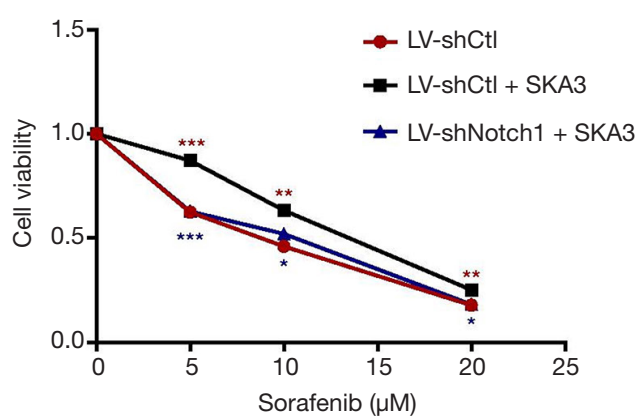

Figure 6 SKA3 promoted the stem cell-like properties through the Notch signaling pathway. We knocked down the expression of Notch1 in SKA3 cells via lentivirus transfection. (A) Transwell migration and invasion assays were performed in indicated cells. The crystal violet was used for dyeing (scale bar: $200 \mu \mathrm{m}$ ). (B) Representative images of colony formation assay in indicated cells. The crystal violet was used for dyeing. (C) Representative images of spheres and histogram analyses in indicated cells (scale bar: $200 \mu \mathrm{m}$ ). (D) CCK8 toxic assay showed that the ability of cell resistance in indicated cells. For the statistical analysis (Student's $t$-test), ${ }^{*}, \mathrm{P}<0.05 ;{ }^{* *}, \mathrm{P}<0.01$; ${ }^{* *}, \mathrm{P}<0.001$. CCK8, cell counting kit-8; SKA3, spindle and kinetochore-associated complex subunit 3.

and associated with poor overall survival of HCC patients. We also demonstrated that SKA3 promoted HCC stem cell-like properties of self-renewal, proliferation, migration, invasion, resistance to Sorafenib, and tumorigenic capacities. Finally, our results showed that SKA3 promoted
HCC stem cell-like characteristics via the Notch signaling pathway.

The SKA complex, which comprises 3 subunits (i.e., SKA1, SKA2, and SKA3), plays a vital role in cell mitosis. Previous study has shown that the complex is related to 
various tumors $(12,13,16)$. In this study, we systematically examined the relationship between each subunit of the SKA complex, and the clinical outcomes, and the malignant biological behavior in HCC. Our results indicated that compared to SKA1 and SKA2, SKA3 was more closely related to clinical outcomes and the stem cell-like properties of HCC.

SKA3 plays an important role in cell mitosis and tumour development. Abad found that SKA3 could interact directly with microtubules to ensure timely mitotic progression (27). The current results highlighted the importance of SKA3, as one subunit of the SKA complex, playing a role in spindle checkpoint silencing and the maintenance of chromosome cohesion in mitosis (28-31). SKA3 has been reported in various cancers, and appears to promote cancers' progression. For example, previous studies have shown that SKA3 promotes cell proliferation and invasion in breast cancer and cervical cancer $(32,33)$. In lung adenocarcinoma, SKA3 was found to promote cell migration through the EGFR-PI3K-Akt axis (34). In addition, Hou et al. showed that SKA3 promoted tumor growth in HCC (15). In this study, we demonstrated that HCC cells overexpressing SKA3 exhibited increased stem cell-like properties of selfrenewal, migration, invasion, proliferation, resistance to Sorafenib and tumorigenic capacities.

To decipher the underlying mechanisms of SKA3 in regulating stem cell-like properties, we evaluated 3 major signaling pathways critical for cancer stemness: Hedgehog, Notch, and Wnt signaling pathways. The results showed that the expression of Notch signaling pathway was correlated with SKA3. Notch signaling pathway is a highly conserved signaling pathway, that plays a key role in the proliferation, self-renewal, differentiation, and apoptosis of cancer cells (35). It is considered as a classical stem-cell pathway, which can maintain and promote the stemness of a variety of cancers (36-39). Our previous studies have demonstrated that the Notch signaling pathway plays a crucial role in promoting the stemness properties of liver cancer stem cells (40-42). In this study, we found that the deletion of Notch1 remarkably inhibited the increasing stem cell-like properties by over-expressing SKA3. Thus, the Notch signaling pathway was shown to play a vital role in promoting the HCC stemness, which was consistent with our previous experimental results. However, the specific sites mediating the association between SKA3 and the Notch signaling pathway are not known. Thus, we intend to investigate the mechanism further and identify the molecular cross-talk. Recombination signal binding protein for immunoglobulin kappa $\mathrm{J}$ region (RBPJ) is a transcription factor that can activate human Notch1 (43). We found that SKA3 was positively correlated with RBPJ (see Figure S5), which may be meaningful in our mechanism research in the future.

The cancer stem cells may provide a therapeutic opportunity to cure and prevent relapse of cancer. Increasingly studies indicated that stem cell specific markers or signaling pathways contribute to maintain and promote the stemness of cancer. Thus, the selective targeting of specific markers and/or signaling pathways is now thought to be an effective therapeutic strategy. Wang et al. demonstrated that CD44 antibody-targeted liposomal nanoparticles, reduced tumor growth and promoted apoptosis by specifically targeting CD44 (44). $\gamma$-secretase inhibitors, a type of Notch inhibitors, have been shown to have antitumor effects and have been subject to clinical trials in cancers (45). In the present study, we showed that SKA3 was positively correlated with CD44 and Notch1. Thus, SKA3, which acted as a regulator of stemness in HCC, might be a potential molecular target for HCC.

\section{Conclusions}

In the present study, we systematically examined the relationship between each subunit of the SKA complex, and the clinical outcomes, and the stem cell-like properties of HCC. The results showed that SKA3 promoted HCC stem cell-like properties via the Notch signaling pathway.

\section{Acknowledgments}

Funding: This work was supported by the National Natural Science Foundation of China (grant numbers 81974380, 81802418) and the Wuhan Science and Technology Bureau (grant numbers 2019020701011431).

\section{Footnote}

Reporting Checklist: The authors have completed the ARRIVE reporting checklist. Available at https://dx.doi. org/10.21037/atm-21-1572

Data Sharing Statement: Available at https://dx.doi. org/10.21037/atm-21-1572

Peer Review File: Available at https://dx.doi.org/10.21037/ atm-21-1572 
Conflicts of Interest: All authors have completed the ICMJE uniform disclosure form (available at https://dx.doi. org/10.21037/atm-21-1572). The authors have no conflicts of interest to declare.

Ethical Statement: The authors are accountable for all aspects of the work in ensuring that questions related to the accuracy or integrity of any part of the work are appropriately investigated and resolved. The study was conducted in accordance with the Declaration of Helsinki (as revised in 2013). The collection and use of human samples were approved by the Ethics Committee of Tongji Hospital, HUST, Wuhan, China (IRB ID: TJ-IRB20210729) and informed consent was taken from all the patients. Animal experiments were approved by the Institutional Animal Care and Use Committee of the HUST (ID: TJH201912001), in compliance with guidelines for the care and use of animals.

Open Access Statement: This is an Open Access article distributed in accordance with the Creative Commons Attribution-NonCommercial-NoDerivs 4.0 International License (CC BY-NC-ND 4.0), which permits the noncommercial replication and distribution of the article with the strict proviso that no changes or edits are made and the original work is properly cited (including links to both the formal publication through the relevant DOI and the license). See: https://creativecommons.org/ licenses/by-nc-nd/4.0/.

\section{References}

1. Bray F, Ferlay J, Soerjomataram I, et al. Global cancer statistics 2018: GLOBOCAN estimates of incidence and mortality worldwide for 36 cancers in 185 countries. CA Cancer J Clin 2018;68:394-424.

2. Yang JD, Heimbach JK. New advances in the diagnosis and management of hepatocellular carcinoma. BMJ 2020;371:m3544.

3. Tabrizian P, Jibara G, Shrager B, et al. Recurrence of hepatocellular cancer after resection: patterns, treatments, and prognosis. Ann Surg 2015;261:947-55.

4. Cabillic F, Corlu A. Regulation of Transdifferentiation and Retrodifferentiation by Inflammatory Cytokines in Hepatocellular Carcinoma. Gastroenterology 2016;151:607-15.

5. Nio K, Yamashita T, Kaneko S. The evolving concept of liver cancer stem cells. Mol Cancer 2017;16:4.
6. Jeyaprakash AA, Santamaria A, Jayachandran U, et al. Structural and functional organization of the Ska complex, a key component of the kinetochore-microtubule interface. Mol Cell 2012;46:274-86.

7. Sivakumar S, Janczyk PŁ, Qu Q, et al. The human SKA complex drives the metaphase-anaphase cell cycle transition by recruiting protein phosphatase 1 to kinetochores. Elife 2016;5:12902.

8. Helgeson LA, Zelter A, Riffle M, et al. Human Ska complex and Ndc80 complex interact to form a loadbearing assembly that strengthens kinetochoremicrotubule attachments. Proc Natl Acad Sci U S A 2018;115:2740-5.

9. Janczyk P€, Skorupka KA, Tooley JG, et al. Mechanism of Ska Recruitment by Ndc80 Complexes to Kinetochores. Dev Cell 2017;41:438-449.e4.

10. Welburn JP, Grishchuk EL, Backer CB, et al. The human kinetochore Ska1 complex facilitates microtubule depolymerization-coupled motility. Dev Cell 2009;16:374-85.

11. Li J, Xuan JW, Khatamianfar V, et al. SKA1 overexpression promotes centriole over-duplication, centrosome amplification and prostate tumourigenesis. J Pathol 2014;234:178-89.

12. Li T, Liu X, Xu B, et al. SKA1 regulates actin cytoskeleton remodelling via activating $\mathrm{Cdc} 42$ and influences the migration of pancreatic ductal adenocarcinoma cells. Cell Prolif 2020;53:e12799.

13. Dou D, Ren X, Han M, et al. Circ_0008039 supports breast cancer cell proliferation, migration, invasion, and glycolysis by regulating the miR-140-3p/SKA2 axis. Mol Oncol 2021;15:697-709.

14. Gao W, Zhang Y, Luo H, et al. Targeting SKA3 suppresses the proliferation and chemoresistance of laryngeal squamous cell carcinoma via impairing PLK1-AKT axismediated glycolysis. Cell Death Dis 2020;11:919.

15. Hou $Y$, Wang $Z$, Huang $S$, et al. SKA3 Promotes tumor growth by regulating CDK2/P53 phosphorylation in hepatocellular carcinoma. Cell Death Dis 2019;10:929.

16. Chen C, Guo Q, Song Y, et al. SKA1/2/3 serves as a biomarker for poor prognosis in human lung adenocarcinoma. Transl Lung Cancer Res 2020;9:218-31.

17. Liu Y, Jin ZR, Huang X, et al. Identification of Spindle and Kinetochore-Associated Family Genes as Therapeutic Targets and Prognostic Biomarkers in Pancreas Ductal Adenocarcinoma Microenvironment. Front Oncol 2020;10:553536.

18. Luo J, Wang P, Wang R, et al. The Notch pathway 
promotes the cancer stem cell characteristics of CD90+ cells in hepatocellular carcinoma. Oncotarget 2016;7:9525-37.

19. Wang F, Zhou H, Xia X, et al. Activated Notch signaling is required for hepatitis $\mathrm{B}$ virus $\mathrm{X}$ protein to promote proliferation and survival of human hepatic cells. Cancer Lett 2010;298:64-73.

20. Zhu Z, Hao X, Yan M, et al. Cancer stem/progenitor cells are highly enriched in CD133+CD44+ population in hepatocellular carcinoma. Int J Cancer 2010;126:2067-78.

21. Murakami S, Ninomiya W, Sakamoto E, et al. SRY and OCT4 Are Required for the Acquisition of Cancer Stem Cell-Like Properties and Are Potential Differentiation Therapy Targets. Stem Cells 2015;33:2652-63.

22. Kim BR, Na YJ, Kim JL, et al. RUNX3 suppresses metastasis and stemness by inhibiting Hedgehog signaling in colorectal cancer. Cell Death Differ 2020;27:676-94.

23. Fang S, Liu M, Li L, et al. Lymphoid enhancer-binding factor-1 promotes stemness and poor differentiation of hepatocellular carcinoma by directly activating the NOTCH pathway. Oncogene 2019;38:4061-74.

24. Wei CY, Zhu MX, Yang YW, et al. Downregulation of RNF128 activates $W \mathrm{nt} / \beta$-catenin signaling to induce cellular EMT and stemness via CD44 and CTTN ubiquitination in melanoma. J Hematol Oncol 2019;12:21.

25. Cui CP, Wong CC, Kai AK, et al. SENP1 promotes hypoxia-induced cancer stemness by HIF-1 $\alpha$ deSUMOylation and SENP1/HIF-1 $\alpha$ positive feedback loop. Gut 2017;66:2149-59.

26. Yamashita T, Ji J, Budhu A, et al. EpCAM-positive hepatocellular carcinoma cells are tumor-initiating cells with stem/progenitor cell features. Gastroenterology 2009; 136:1012-24.

27. Abad MA, Zou J, Medina-Pritchard B, et al. Ska3 Ensures Timely Mitotic Progression by Interacting Directly With Microtubules and Ska1 Microtubule Binding Domain. Sci Rep 2016;6:34042.

28. Gaitanos TN, Santamaria A, Jeyaprakash AA, et al. Stable kinetochore-microtubule interactions depend on the Ska complex and its new component Ska3/C13Orf3. EMBO J 2009;28:1442-52.

29. Zhang Q, Sivakumar S, Chen Y, et al. Ska3 Phosphorylated by Cdk1 Binds Ndc80 and Recruits Ska to Kinetochores to Promote Mitotic Progression. Curr Biol 2017;27:1477-1484.e4.

30. Theis M, Slabicki M, Junqueira M, et al. Comparative profiling identifies C13orf3 as a component of the Ska complex required for mammalian cell division. EMBO J 2009;28:1453-65.

31. Daum JR, Wren JD, Daniel JJ, et al. Ska3 is required for spindle checkpoint silencing and the maintenance of chromosome cohesion in mitosis. Curr Biol 2009; 19:1467-72.

32. Ruan LW, Li PP, Jin LP. SKA3 Promotes Cell Growth in Breast Cancer by Inhibiting PLK-1 Protein Degradation. Technol Cancer Res Treat 2020;19:1533033820947488.

33. Hu R, Wang MQ, Niu WB, et al. SKA3 promotes cell proliferation and migration in cervical cancer by activating the PI3K/Akt signaling pathway. Cancer Cell Int 2018;18:183.

34. Hu DD, Chen HL, Lou LM, et al. SKA3 promotes lung adenocarcinoma metastasis through the EGFR-PI3K-Akt axis. Biosci Rep 2020;40:BSR20194335.

35. Artavanis-Tsakonas S, Rand MD, Lake RJ. Notch signaling: cell fate control and signal integration in development. Science 1999;284:770-6.

36. Vinson KE, George DC, Fender AW, et al. The Notch pathway in colorectal cancer. Int J Cancer 2016;138:1835-42.

37. Pajuelo-Lozano N, Alcalá S, Sainz B Jr, et al. Targeting MAD2 modulates stemness and tumorigenesis in human Gastric Cancer cell lines. Theranostics 2020;10:9601-18.

38. Xiao W, Gao Z, Duan Y, et al. Notch signaling plays a crucial role in cancer stem-like cells maintaining stemness and mediating chemotaxis in renal cell carcinoma. J Exp Clin Cancer Res 2017;36:41.

39. Lin Q, Chen X, Meng F, et al. ASPH-notch Axis guided Exosomal delivery of Prometastatic Secretome renders breast Cancer multi-organ metastasis. Mol Cancer 2019;18:156.

40. Wang X, Wang R, Bai S, et al. Musashi2 contributes to the maintenance of CD44v6+ liver cancer stem cells via notch1 signaling pathway. J Exp Clin Cancer Res 2019;38:505.

41. Xiong S, Wang R, Chen Q, et al. Cancer-associated fibroblasts promote stem cell-like properties of hepatocellular carcinoma cells through IL-6/STAT3/ Notch signaling. Am J Cancer Res 2018;8:302-16.

42. Wang R, Li Y, Tsung A, et al. iNOS promotes CD24+CD133+ liver cancer stem cell phenotype through a TACE/ADAM17-dependent Notch signaling pathway. Proc Natl Acad Sci U S A 2018;115:E10127-36.

43. Lu FM, Lux SE. Constitutively active human Notch1 binds to the transcription factor CBF1 and stimulates transcription through a promoter containing a CBF1responsive element. Proc Natl Acad Sci U S A 
1996;93:5663-7.

44. Wang L, Su W, Liu Z, et al. CD44 antibody-targeted liposomal nanoparticles for molecular imaging and therapy of hepatocellular carcinoma. Biomaterials 2012;33:5107-14.

Cite this article as: Bai $S$, Chen $W$, Zheng $M$, Wang $X$, Peng W, Zhao Y, Wang Y, Xiong S, Cheng B. Spindle and kinetochore-associated complex subunit 3 (SKA3) promotes stem cell-like properties of hepatocellular carcinoma cells through activating Notch signaling pathway. Ann Transl Med 2021;9(17):1361. doi: 10.21037/atm-21-1572
45. Yuan $\mathrm{X}, \mathrm{Wu} \mathrm{H}, \mathrm{Xu} \mathrm{H}$, et al. Notch signaling: an emerging therapeutic target for cancer treatment. Cancer Lett 2015;369:20-7.

(English Language Editors: L. Huleatt and J. Chapnick) 

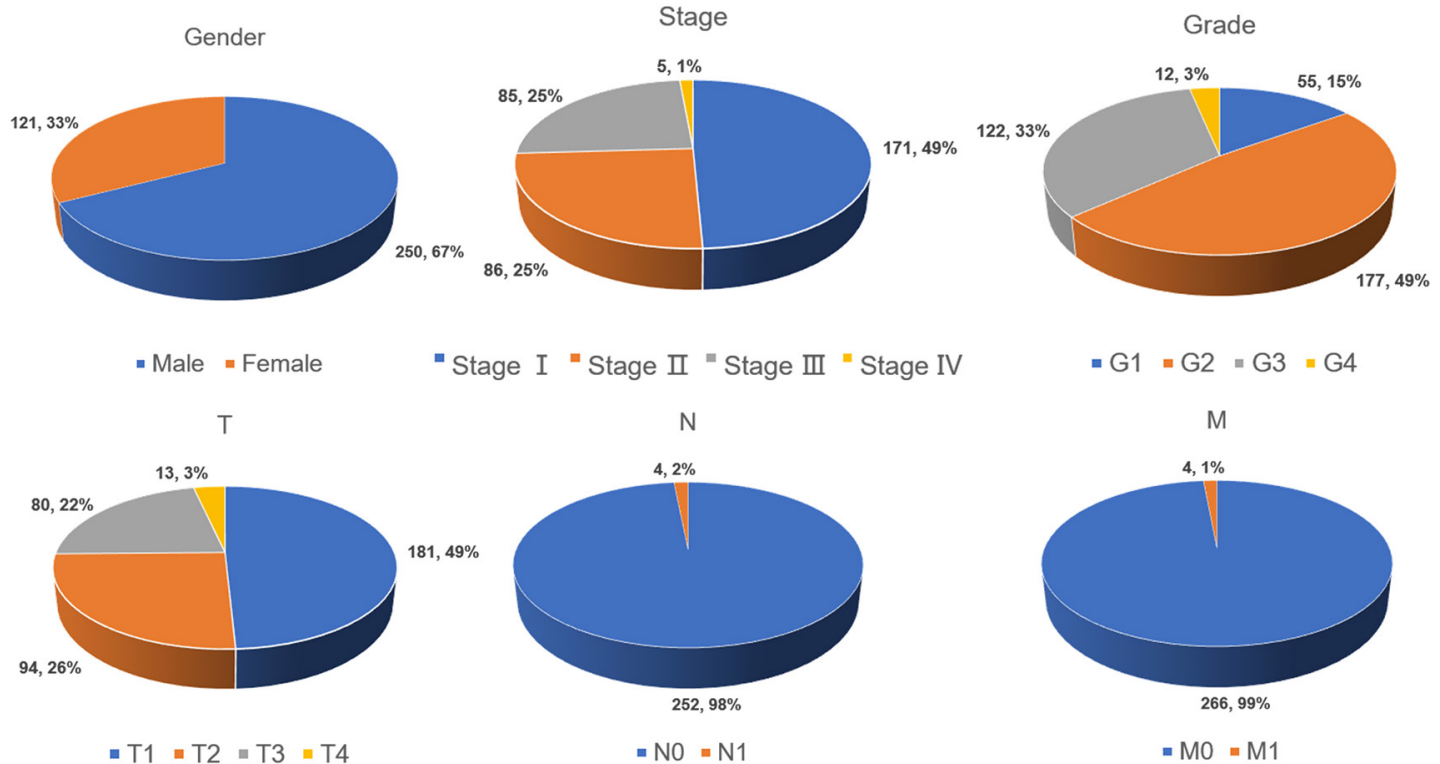

Figure S1 The clinical data of the 374 HCC samples in TCGA LIHC datasets. HCC, hepatocellular carcinoma; TCGA, The Cancer Genome Atlas; LIHC, liver hepatocellular carcinoma.

A

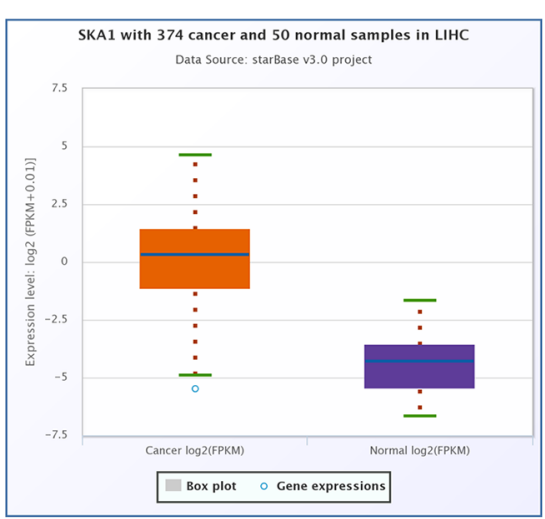

SKA1 (220134)

B

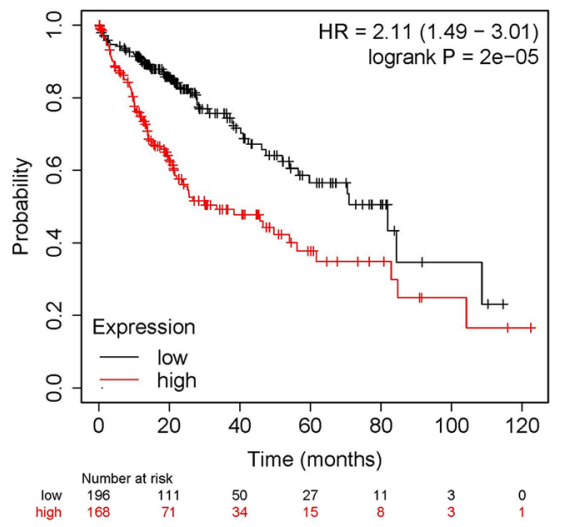

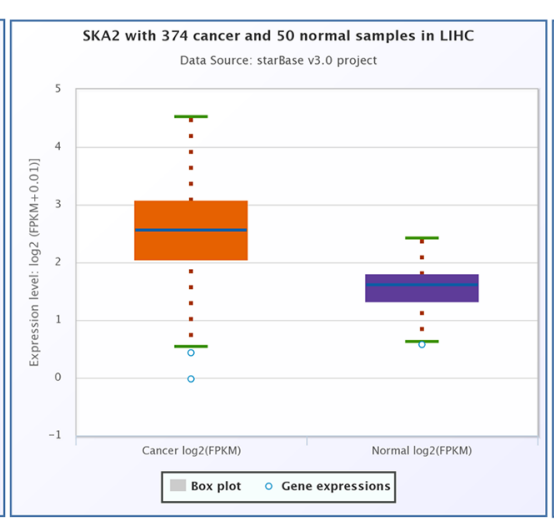

SKA2 (348235)

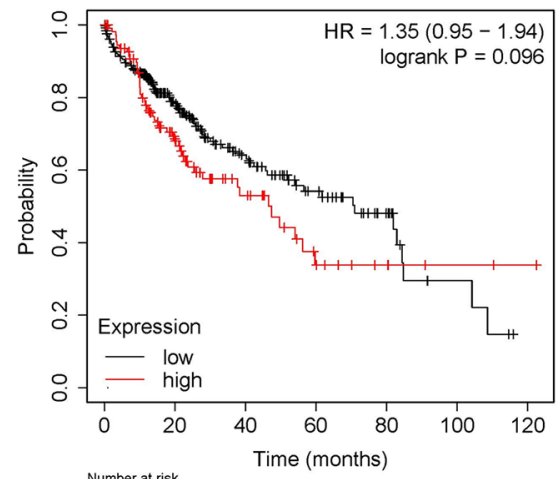

$\begin{array}{lll}\text { low } & \text { Number at risk } \\ \text { high } & 252 & 128 \\ \text { 112 } & 54\end{array}$

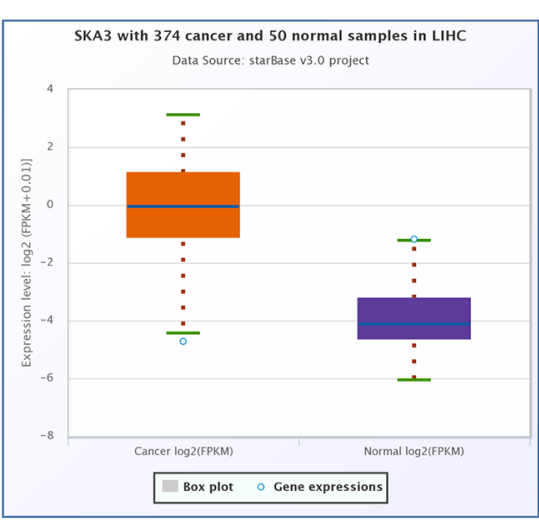

SKA3 (221150)

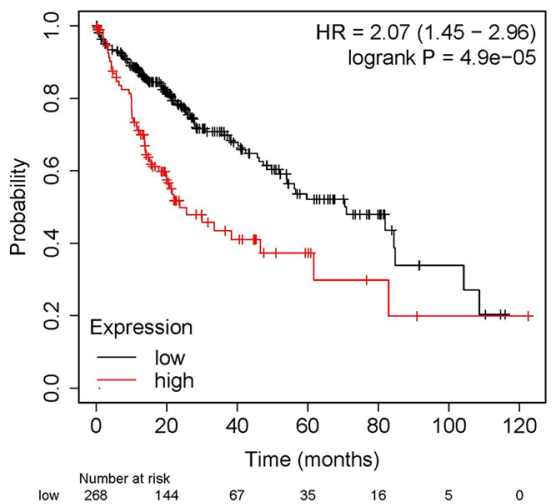

Figure S2 The SKA family was highly expressed in HCC. (A) The expression level of SKA1, SKA2 and SKA3 in the normal liver samples $(\mathrm{n}=50)$ and the HCC samples ( $\mathrm{n}=374)$, as indicated by the TCGA LIHC data set. (B) Kaplan-Meier overall survival curves of patients with HCC in the TCGA cohort (log-rank test). SKA, spindle and kinetochore-associated; SKA1, spindle and kinetochore-associated complex subunit 1; SKA2, spindle and kinetochore-associated complex subunit 2; SKA3, spindle and kinetochore-associated complex subunit 3; HCC, hepatocellular carcinoma; TCGA, The Cancer Genome Atlas; LIHC, liver hepatocellular carcinoma. 
A
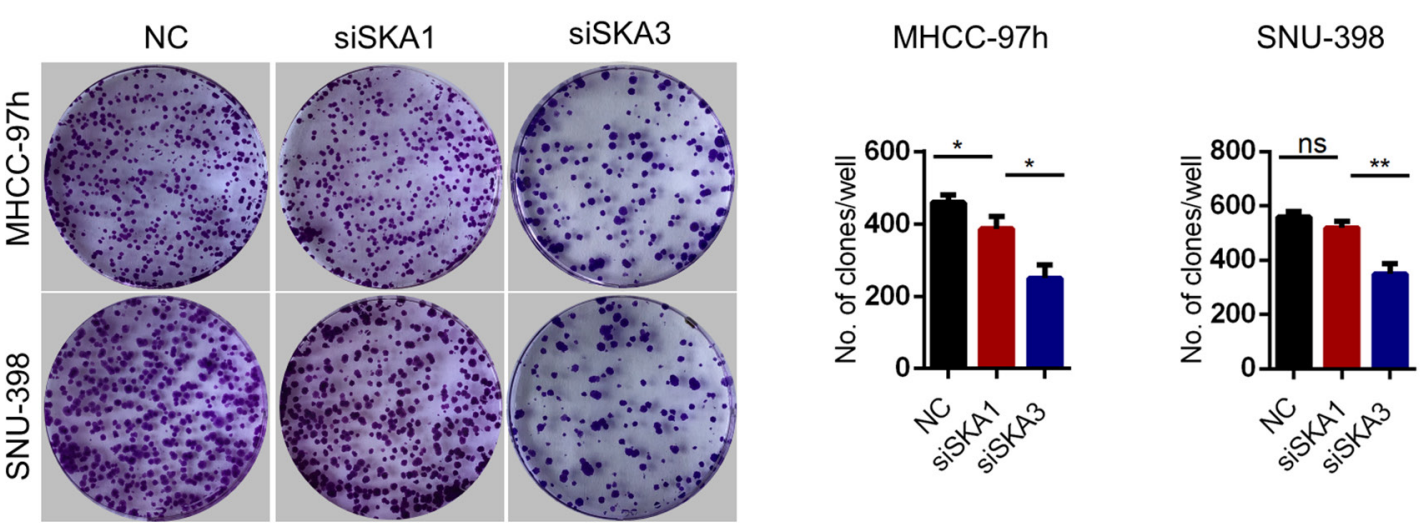

B

NC

siSKA1

siSKA3

MHCC-97h

SNU-398
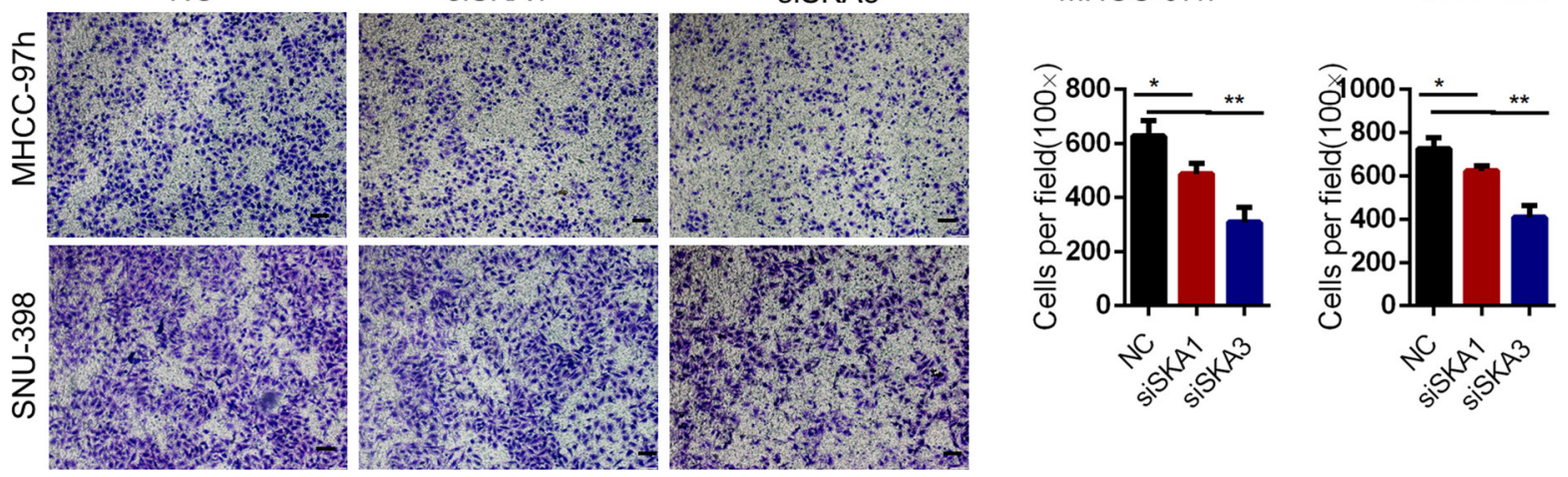

Figure S3 SKA3 had stronger effects on proliferation and migration than SKA1 in HCC. (A) Representative images of colony formation assay in indicated cells staining with crystal violet solution. (B) Representative images of Transwell migration assay in indicated cells staining with crystal violet solution (scale bar: $200 \mu \mathrm{m}$ ). For the statistical analysis (Student's $t$-test), ${ }^{*}, \mathrm{P}<0.05 ;{ }^{* *}, \mathrm{P}<0.01$. ns, not significant; SKA1, spindle and kinetochore-associated complex subunit 1; SKA3, spindle and kinetochore-associated complex subunit 3; HCC, hepatocellular carcinoma.
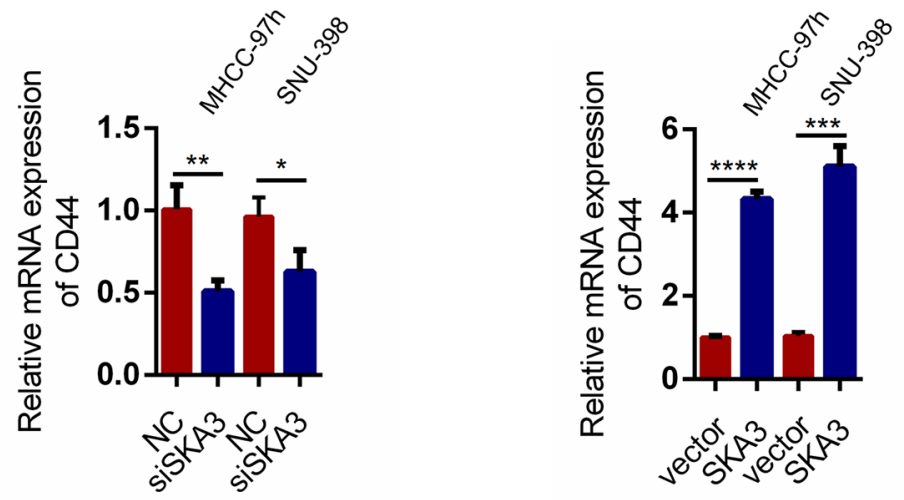

Figure S4 The expression of CD44 were tested by qRT-PCR in indicated cells. For the statistical analysis (Student's $t$-test), *, P<0.05; **, $\mathrm{P}<0.01$; ${ }^{* * *}, \mathrm{P}<0.001$; ${ }^{* * *}, \mathrm{P}<0.0001$. SKA3, spindle and kinetochore-associated complex subunit 3; qRT-PCR, quantitative reverse transcription-polymerase chain reaction. 


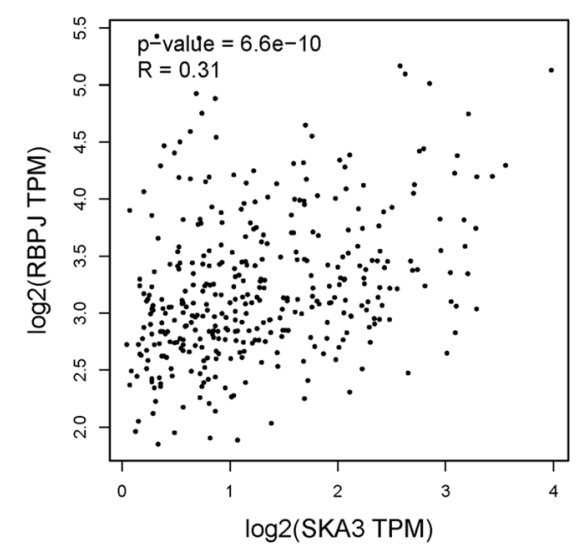

Figure S5 Correlations between RBPJ and SKA3 in TCGA HCC tumor are denoted with Pearson's correlation coefficients. SKA3, spindle and kinetochore-associated complex subunit 3; TCGA, The Cancer Genome Atlas; HCC, hepatocellular carcinoma; TPM, transcript per million. 